This document is a manuscript version of the following article:

Title: An enhanced and validated performance and cavitation prediction model for horizontal axis tidal turbines

By: Kaufmann, Nicholas; Carolus, Thomas; Starzmann, Ralf

Published in: International Journal of Marine Energy

Volume 19, September 2017, Pages 145-163

Publisher: Elsevier | Year: 2017

DOI: 10.1016/j.ijome.2017.07.003

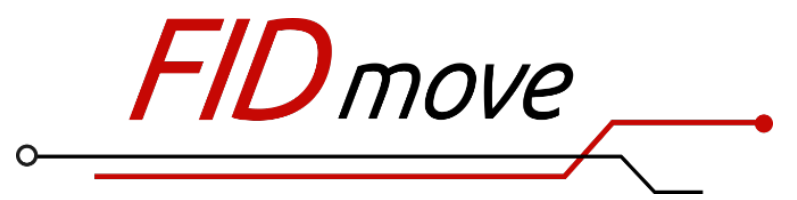

Brought to you by the Scientific Information Service for Mobility and Transport Research

Fachinformationsdienst Mobilitäts- und Verkehrsforschung

Website: www.fid-move.de

Repository: publish.fid-move.de

Contact: publish@fid-move.de

(C) 2017. This manuscript version is made available under the CC-BYNC-ND 4.0 license.

http://creativecommons.org/licenses/by-nc-nd/4.0/ 


\title{
An Enhanced and Validated Performance and Cavitation Prediction Model for Horizontal Axis Tidal Turbines
}

\author{
N. Kaufmann ${ }^{1}$, T.H. Carolus ${ }^{1}$, R. Starzmann ${ }^{2}$ \\ ${ }^{1}$ Institute for Fluid- and Thermodynamics, University of Siegen. 57068 Siegen, Germany \\ ${ }^{2}$ SCHOTTEL HYDRO GmbH, Mainzer Straße 99, 56322 Spay, Germany
}

Corresponding author: nicholas.kaufmann@uni-siegen.de

\begin{abstract}
Tidal energy represents a promising resource for the future energy mix. For harnessing tidal currents free stream horizontal axis turbines have been investigated for some years. The acting physics is very similar to the one of horizontal axis wind turbines, with the additional phenomenon of cavitation, which causes performance reduction, flow induced noise and severe damages to the turbine blade and downstream structures.

The paper presents an enhanced semi-analytical model that allows the prediction of the performance characteristics including cavitation inception of horizontal axis tidal turbines. A central component is the wellknown blade element momentum theory which is refined by various submodels for hydrofoil section lift and drag as a function Reynolds number and angle of attack, turbine thrust coefficient, blade hub and tip losses and cavitation. Moreover, the model is validated by comparison with comprehensive experimental data from two different turbines.

Predicted power and thrust coefficient characteristics were found to agree well with the experimental results for a wide operational range and different inflow velocities. Discrepancies were observed only at low tip speed ratios where major parts of the blades operate under stall conditions. The predicted critical cavitation number is somewhat larger than the measured, i.e. the prediction is conservative. As an overall conclusion the semi-analytical model developed seems to be so fast, accurate and robust that it can be integrated in a future workflow for optimizing tidal turbines.
\end{abstract}

Keywords - Tidal turbine, free stream, cavitation, blade element momentum theory, experimental validation

\section{Nomenclature}

$A \quad$ swept area of the turbine rotor $\left[\mathrm{m}^{2}\right]$

$C_{D} \quad$ drag coefficient [-]

$C_{L} \quad$ lift coefficient [-]

$C_{P} \quad$ power coefficient [-]

$C_{p} \quad$ pressure coefficient [-]

$C_{T} \quad$ thrust coefficient [-]

$D$ drag force $[\mathrm{N}]$

$F \quad$ tip loss correction factor by GLAUERT/PRANDTL [-] factor by

$F_{l} \quad$ tip loss correction factor by SHEN [-]

$F_{a x} \quad$ axial force $[\mathrm{N}]$

$F_{u} \quad$ tangential force $[\mathrm{N}]$

$L \quad$ lift force [N]

$N \quad$ number of rotor blades [-]

$P \quad$ power [W]

$Q \quad$ torque $[\mathrm{Nm}]$

Re Reynolds number [-]

$T \quad$ thrust $[\mathrm{N}]$

$a \quad$ axial induction factor [-]

$a^{\prime} \quad$ tangential induction factor [-]

$c_{a x} \quad$ absolute axial velocity $[\mathrm{m} / \mathrm{s}]$

$c_{\text {ref }} \quad$ cavitation reference velocity $[\mathrm{m} / \mathrm{s}]$

$c_{u} \quad$ absolute circumferential velocity $[\mathrm{m} / \mathrm{s}]$

$d_{\text {tip }} \quad$ rotor diameter [m]

g gravity $\left[\mathrm{m} / \mathrm{s}^{2}\right]$

$h_{\text {hub }}$ hub immersion depth [m]

$l \quad$ chord length [m]

$\dot{m} \quad$ mass flow rate $[\mathrm{kg} / \mathrm{s}]$

$n \quad$ rotational speed $[1 / \mathrm{s}]$

$p_{a t m} \quad$ atmospheric pressure [Pa]

$p_{\text {ref }} \quad$ cavitation reference pressure $[\mathrm{Pa}]$

$p_{v} \quad$ vapour pressure $[\mathrm{Pa}]$

$r \quad$ radius [m]

$u \quad$ circumferential velocity $[\mathrm{m} / \mathrm{s}]$

$w \quad$ relative velocity $[\mathrm{m} / \mathrm{s}]$

$\alpha \quad$ angle of attack $\left[{ }^{\circ}\right]$

$\beta \quad$ flow angle $\left[{ }^{\circ}\right]$

$\gamma \quad$ twist angle $\left[{ }^{\circ}\right]$

$\lambda \quad$ tip speed ratio [-]

$v \quad$ kin. viscosity $\left[\mathrm{m}^{2} / \mathrm{s}\right]$

$\rho \quad$ density $\left[\mathrm{kg} / \mathrm{m}^{3}\right]$

$\sigma \quad$ cavitation number [-]

$\Omega \quad$ angular velocity $[\mathrm{rad} / \mathrm{s}]$

Subscripts

0 position far upstream

1 position before rotor

2 position after rotor

3 position far downstream

0.7 referring to $r / r_{t i p}=0.7$

$\infty \quad$ position in rotor plane

DF $\quad$ based on dynamic forces 
M based on momentum conservation

crit critical value

Abbreviations

hub at hub

BE blade element

max maximum

BEM blade element momentum theory

$\min$ minimum

tip at blade tip

Korrekturen basierend auf den Anmerkungen von Reviewer 1

Korrekturen basierend auf den Anmerkungen von Reviewer 2 sonstige Korrekturen 


\section{$1 \quad$ Introduction}

Tidal energy represents a promising resource for the future energy mix. It offers a superb predictability and hence can act as a reliable supplement to other renewable energy resources like wind or solar radiation. For harnessing tidal currents free stream horizontal axis turbines have been investigated for some years. The acting physics is very similar to the one of horizontal axis wind turbines, with the additional phenomenon of cavitation. Cavitation contributes to performance reduction, flow induced noise and can cause severe damages to the turbine blade and downstream structures [1].

Advanced hydraulic design methods of stream turbines, possibly with an integrated optimization, require the prediction of the hydraulic performance characteristics for the entire operating range of a given turbine design. In principle this can be achieved by a full numerical simulation of the flow in the turbine, e.g. utilizing a Reynolds-averaged Navier-Stokes method. Yet, this is computationally rather expensive, especially since the computational domain must be extremely large for free stream turbines. An alternative is a semianalytical method, for instance based on the well-known blade element momentum theory (BEM). The computational cost is low, but the initial efforts for modeling and validation are by far not negligible. Since the strategy of our future research is the utilization of advanced optimization methods with their typically large number of evaluation steps, we focus on the latter approach.

BEM has been used intensively for blade design particularly in the field of wind turbines and turbine performance prediction (see e. g. GASCH and TWELE [2], HANSEN [3], BURTON [4], GERHARD et al. [5], NREL's time-domain wind turbine aerodynamics module AeroDyn [6]). Numerous empirical correlations were developed to improve its accuracy and extend its range of validity; e.g. for the thrust (see e.g. GLAUERT [7], SPERA [8] and BUHL [9] or the losses at the blade root and tip (GLAUERT [7], SHEN et al. [10]). During the last decade a few applications of BEM to tidal turbines have been published, (BATTEN et al.[11], MASTERS et al. [12]). Based on the cavitation tests with 2D airfoil sections by MOLLAND et al. [13], a first preliminary cavitation model was integrated into BEM by BATTEN et al. [14]. He was able predicting the cavitation inception along the span of a tidal turbine at a selected operating point while varying the pitching angle. The pressure equalisation at the blade tip was not taken into account. BUCKLAND et al [] extended this approach to by including a varying azimuth angle. the BALTAZAR et al. [15] presented a model for predicting the cavitation number for a wide range of operating points based on the pressure distribution of the blade. This model took into account various rotor rotational speeds, but the effect of the near-tip flow on the pressure distribution has not been addressed. Moreover, an experimental validation has not been published. BAL et al. used in [16] a vortex lattice method to predict the cavitation propagation along a tidal turbine blade and qualitatively compared the results to experiments. A quantitative determination of the cavitation inception over wide range of operating points, modeled or measured, has not been presented.

The objective of this paper is to present a BEM model, enhanced by a number of submodels for the accurate prediction of cavitation inception and off design operating points. Moreover, emphasis is laid on a comprehensive validation by either comparison with published experimental data or new measurements.

\section{Turbine Performance Prediction Model}

In this section we shortly summarize the basic BEM and explain the auxiliary semi-empirical models used to include losses, off-design and cavitation. A widely used representation of tidal turbine performance and cavitation characteristics are shown schematically in Fig. 1 in terms of the non-dimensional quantities - tip speed ratio

$$
\lambda \equiv \frac{u_{t i p}}{c_{0}}
$$

- power coefficient

$$
C_{P} \equiv \frac{P}{0.5 \rho c_{0}^{3} A},
$$

- thrust coefficient

$$
C_{T} \equiv \frac{F_{a x}}{0.5 \rho c_{0}^{2} A}
$$


- cavitation number

$$
\sigma=\frac{p_{r e f}-p_{v}}{0.5 \rho c_{r e f}^{2}},
$$

$P$ is the shaft power available from the turbine, $F_{a x}$ the thrust on the rotor. The tip speed is obtained from the rotor diameter and its rotational speed $u_{t i p}=r_{t i p} \Omega=\pi d_{t i p} n, A=\pi / 4 d_{t i p}^{2}$ is the swept area of the rotor and $c_{0}$ the free flow velocity far upstream of the turbine. $p_{v}$ is the vapor pressure of the fluid and $p_{\text {ref }}$ and $c_{\text {ref }}$ a representative static pressure and velocity related to the turbine flow field. Cavitation is avoided when the cavitation number exceeds a critical value. E.g. in case of a pipe flow, where the static pressure approaches the vapor pressure, cavitation inception is associated with $\sigma=\sigma_{\text {crit }}=0$.
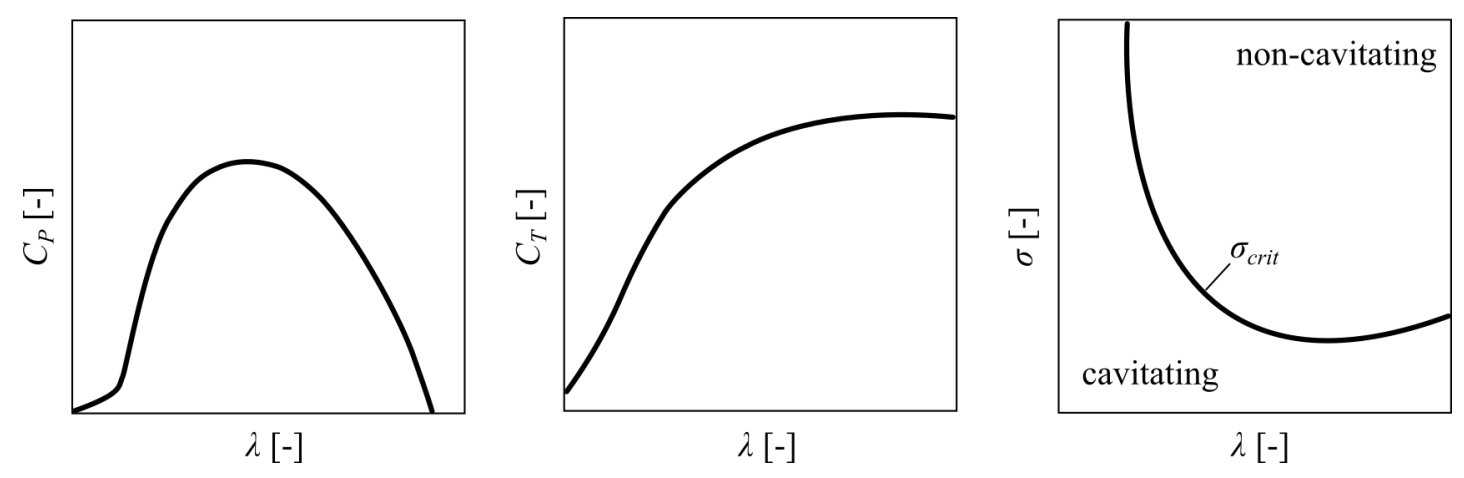

Fig. 1: Non-dimensional turbine characteristics: Power coefficient, thrust coefficient and critical cavitation number as a function of tip-speed ratio (schematically)

\subsection{Blade Element Momentum Theory (BEM)}

The extraction of energy by the turbine causes a retardation of the free flow velocity $c_{0}$ far upstream to $c_{3}$ far downstream. Mass conservation forces the stream tube in Fig. 2 to expand from up- to downstream.

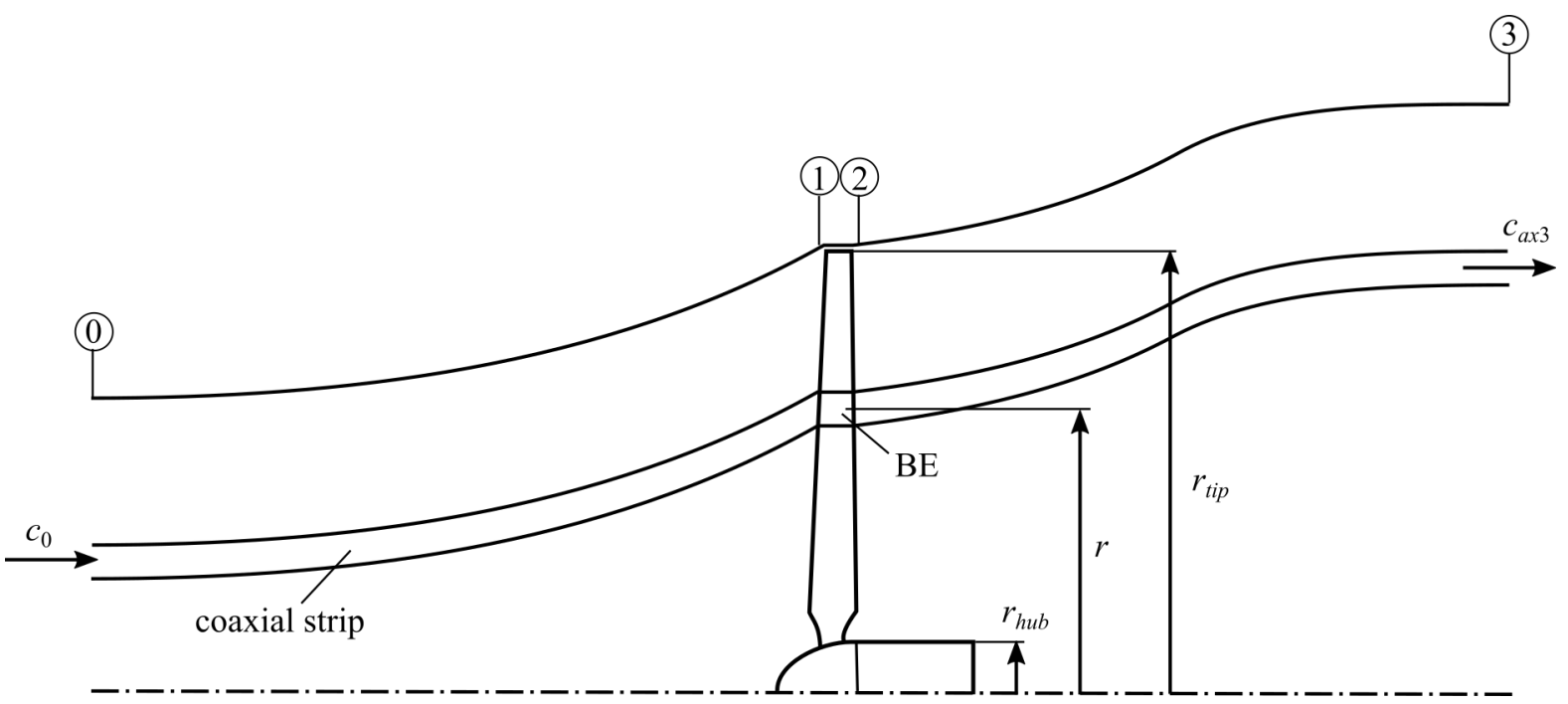

Fig. 2: Horizontal free flow turbine in boundary stream tube [5]

The velocity $c_{3}$ has an axial component $c_{a x 3}$ and a circumferential (or swirl) component $c_{u 3}$. The stream tube can be segmented into a number of coaxial strips each containing blade elements (BE) of the radial extension $\delta r$. In each coaxial strip the velocities are assumed to be not a function of the radius. Hence, the velocity triangles as in Fig. 3 apply. We further assume that (i) the fluid is incompressible, (ii) the inflow has no swirl and (iii) the stream lines in the rotor are parallel to the axis of the rotor. Hence the absolute velocity of the fluid entering the 
rotor plane $c_{1}$ equals its axial component $c_{a x 1}$, and $c_{a x 2}=c_{a x 1}$. The so called axial induction factor $a$ is commonly used to describe the meridional velocity in the rotor plane

$$
c_{a x 1}=c_{a x 2}=c_{0}(1-a) \text { and } c_{a x 3}=c_{0}(1-2 a) \text {. }
$$

In the exit plane of the rotor the velocity in the absolute frame of reference has the meridional and circumferential components $c_{a x 2}$ and $c_{u 2}$, respectively. Similar to the axial induction factor, a circumferential induction factor $a^{\prime}$ is defined such that

$$
c_{u 2}=-2 a^{\prime} u
$$

with the circumferential velocity $u=r \Omega=2 \pi r n$.

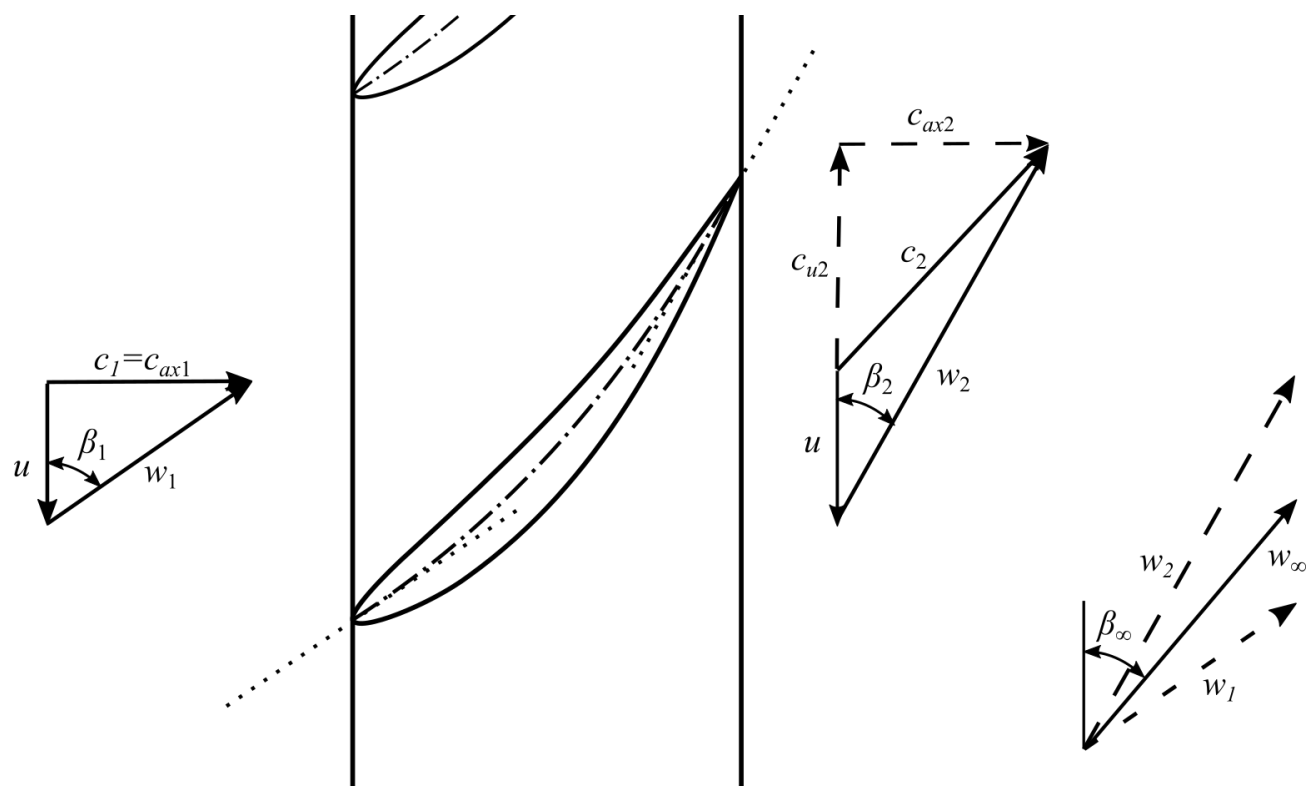

Fig. 3: Velocity triangles at the rotor entrance and exit planes, after [5]

The conservation of axial momentum on the coaxial strip, from far upstream to downstream, yields the axial force acting on the rotor segment within the coaxial strip

$$
\delta F_{a x, M}=\left(c_{0}-c_{a x 3}\right) \delta \text { R\& }=4 a(1-a) c_{0}^{2} \rho \pi r \delta r .
$$

The mass flow rate through a bladed annulus of $\delta A=2 \pi r \delta r$ then becomes

$$
\delta r \&=\rho c_{a x 1} \delta A=\rho c_{0}(1-a) 2 \pi r \delta r .
$$

The extracted power is obtained via EULER'S turbine equation as

$$
\delta P_{M}=u\left(c_{u 2}-c_{u 1}\right) \delta r \&=4 \Omega^{2} r^{3} a^{\prime}(1-a) c_{0} \rho \pi \delta r .
$$

Introducing the local-speed ratio

$$
\lambda_{r} \equiv \frac{u}{c_{0}}=\frac{r}{r_{\text {tip }}} \lambda
$$

the local thrust and power coefficients become

$$
C_{T, M}=\frac{\delta F_{a x, M}}{\rho c_{0}^{2} \pi r \delta r}=4 a(1-a)
$$


and

$$
C_{P, M}=\frac{\delta P_{M}}{\rho c_{0}^{3} \pi r \delta r}=4 a^{\prime}(1-a) \lambda_{r}^{2},
$$

respectively.

For any given value of $\lambda_{r}$ the two eqs. (2.11 and 2.12) bear the four unknown quantities $C_{T, M}, C_{P, M}, a$ and $a^{\prime}$. So far, frictional losses of the rotor blades have not been included. Additional equations are obtained by considering each $\mathrm{BE}$ as an isolated hydrofoil segment experiencing a lift and a drag force.

As common in the field of low solidity axial turbomachinery, the vector mean of the velocities $w_{1}$ and $w_{2}$ in the rotating frame of reference (see Fig. 3) is assumed to be the relevant velocity for the hydrodynamic forces:

$$
\stackrel{\text { ư }}{w_{\infty}}=\frac{1}{2}\left(\stackrel{\text { ur }}{w_{1}}+\underset{w_{2}}{w_{2}}\right) .
$$

Implementing the previous relationships in eq. (2.13) one obtains the magnitude

$$
w_{\infty}=\frac{1}{2} \sqrt{4 c_{a x}^{2}+\left(2 u-c_{u 2}\right)^{2}}=c_{0} \sqrt{(1-a)^{2}+\left(1+a^{\prime}\right)^{2} \lambda_{r}^{2}} .
$$

and the angle between the flow direction and rotor (flow angle)

$$
\beta_{\infty}=\arctan \left(\frac{2 c_{a x}}{2 u-c_{u 2}}\right)=\arctan \left(\frac{1-a}{1+a^{\prime}} \frac{1}{\lambda_{r}}\right),
$$
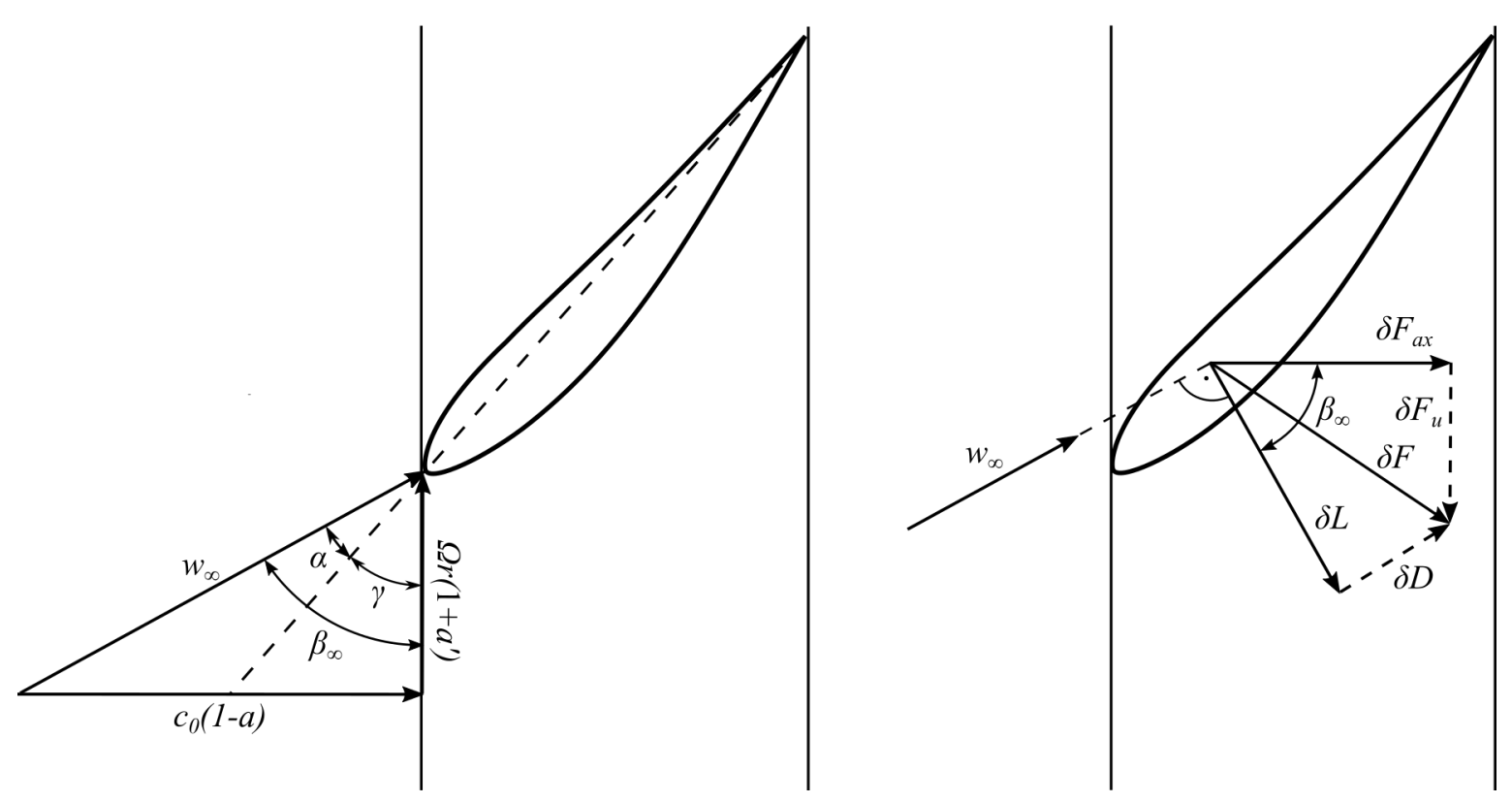

Fig. 4: Flow velocities (left) and resulting dynamic forces (right) on blade element

The axial and circumferential (i.e. in plane) dynamic forces acting on a BE can be expressed in terms of lift $L$ and drag $D$ and the flow angle $\beta_{\infty}$ as

$$
\begin{aligned}
& \delta F_{a x, D F}=\delta L \cos \left(\beta_{\infty}\right)+\delta D \sin \left(\beta_{\infty}\right), \\
& \delta F_{u, D F}=\delta L \sin \left(\beta_{\infty}\right)-\delta D \cos \left(\beta_{\infty}\right),
\end{aligned}
$$

respectively. 
We now replace $L$ and $D$ in eqs. (2.16) and (2.17) by the lift and drag coefficient $C_{L}$ and $C_{D}$. Due to their customary definitions $\mathrm{BE}$ chord length $l$ and radial extension $\delta r$ as well as the number of blades $N$ come into play:

$$
\begin{aligned}
& \delta F_{a x, D F}=N \frac{1}{2} \rho w_{\infty}^{2}\left(C_{L} \cos \beta_{\infty}+C_{D} \sin \beta_{\infty}\right) l \delta r \\
& \delta F_{u, D F}=N \frac{1}{2} \rho w_{\infty}^{2}\left(C_{L} \sin \beta_{\infty}-C_{D} \cos \beta_{\infty}\right) l \delta r,
\end{aligned}
$$

For a given type of hydrofoil $C_{L}$ and $C_{D}$ are primarily a function the angle of attack $\alpha$ of the flow to the hydrofoil section, and - as discussed later - the local Reynolds number. The hydrofoil section is placed in the rotor plane under a local twist angle $\gamma$. (Note that $\gamma$ stays the same at any time for a fixed pitch turbine.) Hence, the resulting angle of attack $\alpha$ of the flow to the hydrofoil section is

$$
\alpha=\beta_{\infty}-\gamma
$$

As for any low-solidity blade cascade, the flow field around a BE is assumed not to be affected by its circumferential neighbors. Hence, in this rudimental standard hydrofoil polars for isolated hydrofoils can be utilized to get $C_{L}$ and $C_{D}$. strip is

The extracted power associated with the in-plane forces on all BEs due to dynamic forces in the coaxial

$$
\delta P_{D F}=\delta F_{u, D F} r \Omega
$$

Eventually we obtain the local thrust and power coefficient

$$
C_{T, D F}=\frac{\delta F_{a x, D F}}{\rho c_{0}^{2} \pi r \delta r}=\frac{N l}{2 \pi r}\left((1-a)^{2}+\lambda_{r}^{2}\left(1+a^{\prime}\right)^{2}\right) C_{a x}
$$

and

$$
C_{P, D F}=\frac{\delta P_{D F}}{\rho c_{0}^{3} \pi r \delta r}=\frac{N l}{2 \pi r}\left((1-a)^{2}+\lambda_{r}^{2}\left(1+a^{\prime}\right)^{2}\right) C_{u} \lambda_{r},
$$

where we introduced a normal and circumferential force coefficient

$$
C_{a x} \equiv C_{L} \cos \beta_{\infty}+C_{D} \sin \beta_{\infty}
$$

and

$$
C_{u} \equiv C_{L} \sin \beta_{\infty}-C_{D} \cos \beta_{\infty}
$$

respectively.

Equating $C_{T, M}=C_{T, D F}$ and $C_{P, M}=C_{P, D F}$ from eqs. (2.11), (2.12) and (2.22), (2.23) yields two nonlinear equations for the two unknowns $a$ and $a^{\prime}$ :

$$
\begin{aligned}
& 4 a(1-a)=\frac{N l}{2 \pi r}\left((1-a)^{2}+\left(\frac{r}{r_{\text {tip }}} \lambda\right)^{2}\left(1+a^{\prime}\right)^{2}\right) C_{a x}, \\
& 4 a^{\prime}(1-a)^{2}\left(\frac{r}{r_{\text {tip }}} \lambda\right)=\frac{N l}{2 \pi r}\left((1-a)^{2}+\left(\frac{r}{r_{\text {tip }}} \lambda\right)^{2}\left(1+a^{\prime}\right)^{2}\right) C_{u}
\end{aligned}
$$

Hence, for each tip speed ratio $\lambda$ and BE located at radius $r$, the induction factors $a$ and $a^{\prime}$ can be calculated. For operating conditions or geometries with a large influence of three dimensional effects (e.q. stall), there might be 
no solution of eqs. (26) and (27). Additionally, eq. 2.11 yields non-physical thrust coefficients for axial induction factors exceeding a critical value (see section 2.2.1). Furthermore multi-valued solutions of $a$ and $a^{\prime}$ cannot be precluded, hence the usage of the physically reasonable values must be ensured.

The overall thrust and extracted power coefficients as a function of the tip-speed ratio, i.e. the targeted characteristics of the turbine, are obtained by a summation over all $n$ coaxial strips:

$$
\begin{gathered}
C_{T}(\lambda)\left(\equiv \frac{F_{a x}(\lambda)}{0.5 \rho c_{0}^{2} A}\right)=\frac{\sum_{\text {all BE}} \delta F_{a x, D F}}{0.5 \rho c_{0}^{2} A}=\frac{\sum_{\text {all BE }} C_{T, D F}(\lambda) 2 \pi r \delta r}{A} \\
C_{P}(\lambda)\left(\equiv \frac{P(\lambda)}{0.5 \rho c_{0}^{3} A}\right)=\frac{\sum_{\text {all BE}} \delta P_{D F}}{0.5 \rho c_{0}^{3} A}=\frac{\sum_{\text {all BE }} C_{P, D F}(\lambda) 2 \pi r \delta r}{A}
\end{gathered}
$$

\subsection{Supplementary Semi-empirical Submodels}

\subsubsection{Tip/Hub Loss Models}

In the basic BEM as presented above it was assumed that the flow field around a BE is not to be affected by its spanwise neighbors along the blade. For a blade with its finite length this does not hold true. Especially at the tip a tip vortex develops inducing additional drag. But also the hub region of the blade is affected by vortex type secondary flows.

A customary model for taking the degradation of tip and hub flow into account is due to PRANDTL and GLAUERT [7] (see e.g. a summary in GASCH [2]) and MORIATY and HANSEN [17]). The idea is to introduce in the momentum equations (2.11) and (2.12) a factor $F$ that is a function of the radius $r$ where a BE is located:

$$
\begin{aligned}
& C_{T, M}=F(r) \cdot 4 a(1-a)^{2} \\
& C_{P, M}=F(r) \cdot 4 a^{\prime}(1-a)^{2} \lambda_{r}^{2} .
\end{aligned}
$$

The tip loss factor as proposed by GLAUERT is

$$
F_{t i p}(r)=\frac{2}{\pi} \arccos \left(\exp \left(\frac{N}{2} \frac{r_{t i p}-r}{r \sin \left(\beta_{\infty}\right)}\right)\right) .
$$

It gradually reduces the axial force and power extraction of those BEs close to the blade tip. In a similar way the approach by MORIATY and HANSEN [17] modifies the hub region:

$$
F_{h u b}(r)=\frac{2}{\pi} \arccos \left(\exp \left(\frac{N}{2} \frac{r-r_{h u b}}{r \sin \left(\beta_{\infty}\right)}\right)\right)
$$

Eventually, the two factors are combined into one single [17]

$$
F(r)=F_{t i p}(r) F_{h u b}(r) \text {. }
$$

For consistency, the BE thrust and power coefficients in terms of hydrofoil drag and lift coefficients (eqs. (2.22) and (2.23)) need to be modified as well. SHEN et al. proposed in [18] a correction of the normal and circumferential force coefficients $C_{a x}$ and $C_{u}$. Hence, eqs. (2.22) and (2.23) become

$$
C_{T, D F}=\frac{N l}{2 \pi r}\left((1-a)^{2}+\lambda_{r}^{2}\left(1+a^{\prime}\right)^{2}\right) F_{1} C_{a x}
$$

and

$$
C_{P, D F}=\frac{N l}{2 \pi r}\left((1-a)^{2}+\lambda_{r}^{2}\left(1+a^{\prime}\right)^{2}\right) F_{1} C_{u} \lambda_{r}
$$


Based on their investigations in [10] and further studies of the influence of the tip shape, SHEN et al. proposed in [18] an expression for $F_{1}$ as

$$
F_{1}=\frac{2}{\pi} \arccos \left(\exp \left(-g_{F 1} \frac{N}{2} \frac{r_{t i p}-r}{r_{t i p} \sin \left(\beta_{\infty}\right)}\right)\right)
$$

with

$$
g_{F 1}=\exp \left(-k_{1}\left(N \lambda-k_{2}\right)\right)+0.1
$$

SHEN et al. in [10] set the empirical constants $k_{1}=0.125$ and $k_{2}=21$, obtained from two different wind turbines). Note, that as the number of blades or the tip-speed ratio approaches infinity, $F_{1}$ becomes 1 , and at the blade tip $F_{1}$ is always zero.

\subsubsection{Thrust Correction}

It is well known that axial induction factors $a$ exceeding a critical value $a_{\text {crit }} \approx 1 / 3$ correspond to unrealistic thrust coefficients. As obvious from eq. (2.5) the wake velocity $c_{a x 3}$ even would tend to zero as $a$ approaches 0.5 , with the consequence of an infinitely large diameter of the downstream stream tube.

Based on measurements GLAUERT [7] proposed an empirical parabolic function of $C_{T}(\lambda)$ and $a(\lambda)$ for $a \geq a_{\text {crit }}$. This function was designed to meet the value and slope according to the basic BEM at $a=a_{\text {crit }}$ and yields $C_{T}=2$ as of a solid disc at $a=1$. Alternative empirical functions are given e.g. in HANSEN [3], BURTON [4] or SPERA [8]. GLAUERT's correction does not include the correction factor $F$ for the tip and hub losses. As BUHL [9] pointed out, this causes a discontinuity at the transition from the basic BEM to the empirical relation for $F<1$. This behavior is illustrated in Fig. 5. Therefore BUHL suggested a modification to GLAUERT's model by including $F$ for $a \geq 0.4$ that is used throughout this work:

$$
C_{T, M}=\frac{8}{9}+\left(4 F-\frac{40}{9}\right) a+\left(\frac{50}{9}-4 F\right) a^{2}, a \geq 0.4
$$

Note that for $F=1$ GLAUERT's model is recovered. Fig. 4 shows a comparison of the modified BEM (i.e. including the loss factor $F$ ) and the two empirical models by GLAUERT and BUHL.

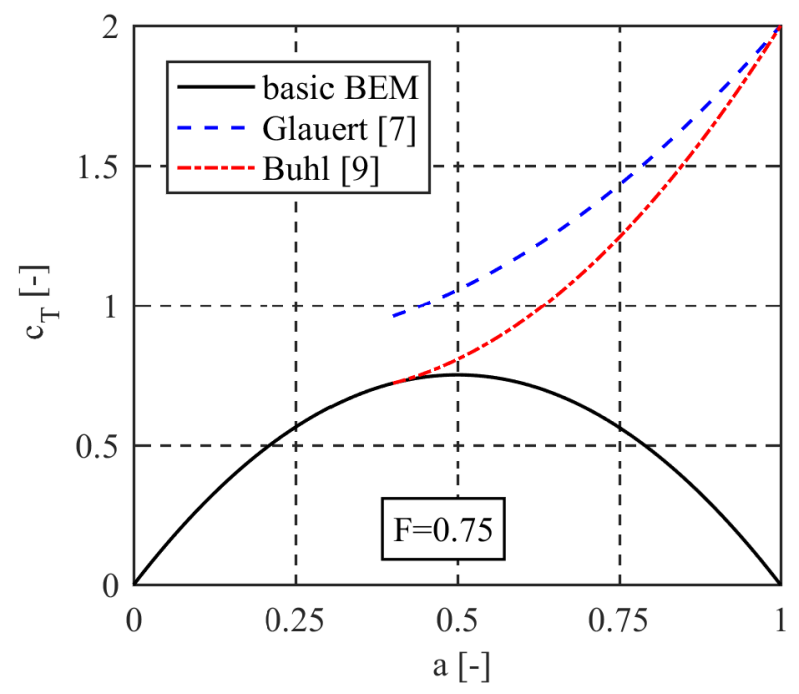

Fig. 5: Comparison of GLAUERTS's and BUHLS's thrust correction for an assumed loss factor $F=0.75$

\subsubsection{Cavitation Model}

Cavitation occurs when the static pressure in a flow field drops below the vapor pressure of the fluid. Cavitation in a turbine is a very complex phenomenon affected by a variety of factors, which naturally can not all be 
accounted for within a semi-analytic BEM. The aim of the presented model is predicting the inception of cavitation in order to determine the turbine operating range free of cavitation as a function of design, placement below the free surface and inflow velocity to the rotor. A performance prediction of the cavitating turbine is by no means an outcome of the targeted model. This is also not of interest since damaging cavitation must strictly be avoided.

We now elaborate on the cavitation number according to eq. (2.4). BALTAZAR et al. [15] chose a reference velocity $c_{r e f}=d_{t i p} n$, whereas we prefer a fictitious flow velocity in the rotating frame of reference

$$
c_{r e f, 0.7} \equiv \sqrt{c_{0}^{2}+\left(r_{0.7} \Omega\right)^{2}}=c_{0} \sqrt{1+(0.7 \lambda)^{2}}
$$

- fictitious, because $c_{0}$ is not in the rotor plane but the free flow velocity $c_{0}$ far upstream. This is a customary choice e.g. by the Potsdam Model Basin (Schiffbau-Versuchsanstalt Potsdam, SVA), where some of the validation tests reported below, had been carried out [19]. The reference pressure is chosen as

$$
p_{\text {ref }}=p_{a t m}+\rho g h_{h u b}
$$

where $p_{a t m}$ is the atmospheric pressure above water level, $\rho g h_{h u b}$ the hydrostatic pressure at the immersion depth of the nacelle and $g$ the gravitational acceleration. Eventually we obtain a working formula for the cavitation number

$$
\sigma_{0.7}=\frac{p_{a t m}+\rho g h_{h u b}-p_{v}}{0.5 \rho c_{0}^{2}\left(1+(0.7 \lambda)^{2}\right)}
$$

For each $\lambda$ the value of $\sigma_{0.7}$, where cavitation inception is observed, is called $\sigma_{0.7, \text { crit. }}$ It is usually determined experimentally in the cavitation tunnel by varying $p_{a t m}$ and presented as shown in Fig. 1. Due to the particular choice of reference quantities, cavitation inception of the turbine is always associated with values of $\sigma_{0.7, \text { crit }}$ larger than zero.

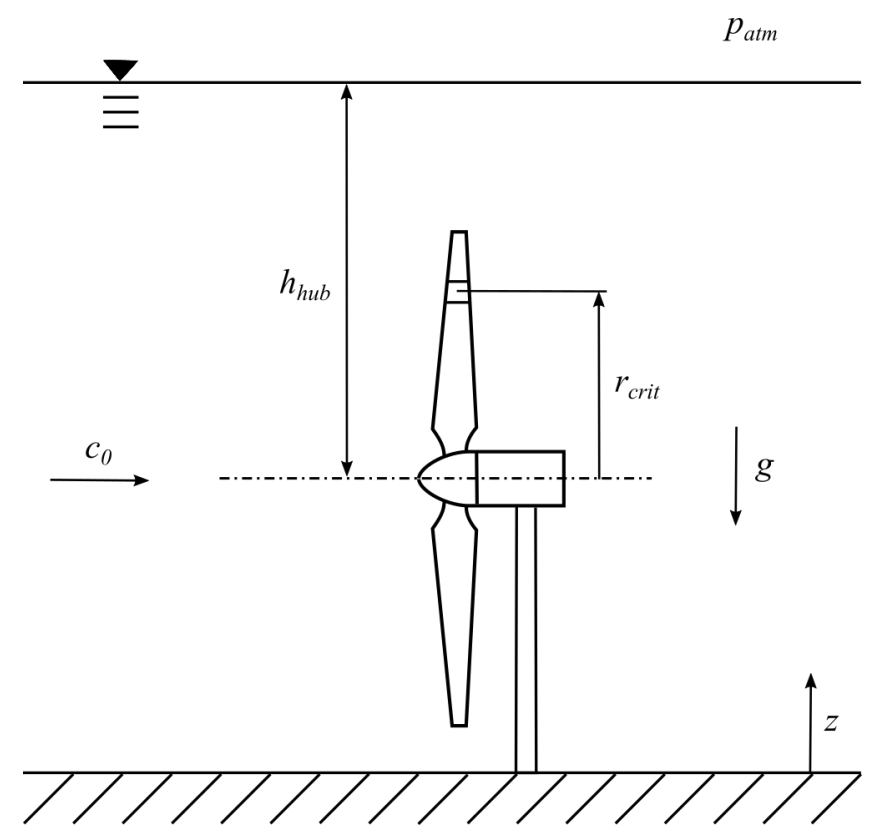

Fig. 6: Tidal turbine in a free stream on support structure (schematically)

Within our modeling the a priori prediction of $\sigma_{0.7, c r i t}(\lambda)$ of the turbine is crucial. Cavitation may occur on the blade suction surface and near the blade tip region due to the presence of the tip vortex. Typically, the onset of tip vortex cavitation is prior to blade suction surface cavitation. Since tip vortex cavitation does rarely harm the blade structure nor degrade the turbine performance, we concentrate on the blade suction surface cavitation. Then, an estimate of the turbine cavitation characteristics can be made on the basis of the chordwise pressure distributions of the BEs which are linked to lift and drag. It is sufficient to consider the critical $\mathrm{BE}_{\text {crit }}$, which encounters cavitation first. Since the hydrostatic pressure in the water decreases with $z$, naively one would expect that $\mathrm{BE}$ to be critical, which is momentarily least immersed, i.e. at the tip of a blade in upright position. 
Because of tip effects, however, the surface pressure differential between pressure and suction side in the tip region of each blade tends to level out and hence the critical suction peak. Therefore, $\mathrm{BE}_{c r i t}$ is located at the a priori unknown distance $r_{\text {crit }}$ from the center of the hub (Fig. 6). coefficient

The surface pressure distribution in chordwise direction of a $\mathrm{BE}$ is known in terms of the pressure

$$
\left.C_{p}\right|_{B E}=\frac{p-p_{\infty}}{0.5 \rho w_{\infty}^{2}}
$$

(see the next section). Here the tip effects are taken into account by replacing $\left.C_{p}\right|_{B E}$ by $\left.F_{1}(r) \cdot C_{p}\right|_{B E}, F_{1}$ being the already introduced force correction from SHEN et al. [18]. The static pressure $p_{\infty}$ far upstream of a BE at radius $r$ is

$$
p_{\infty}=p_{a t m}+\rho g\left(h_{h u b}-r\right) .
$$

Hence,

$$
\left.p_{\min }\right|_{B E}=\left.0.5 \rho w_{\infty}^{2}(r) \cdot F_{1}(r) C_{p, \min }\right|_{B E}+p_{a t m}+\rho g\left(h_{h u b}-r\right)
$$

Coming from a non-cavitation condition, where $p_{\min }>p_{v}$, cavitation inception is equivalent to $p_{\min }=p_{v}$ at least at one $\mathrm{BE}$. $\mathrm{BE}_{\text {crit }}$ is the first among all spanwise $\mathrm{BEs}$, for which $p_{\min }=p_{v}$. This eventually allows identifying $r_{c r i t}$. Evaluating $\left.p_{\min }\right|_{B E_{c r i t}}=p_{v}$ yields the nominator in eq. (2.42) exclusively in terms of blade related quantities

$$
p_{a t m}+\rho g h_{h u b}-p_{v}=\rho g r_{c r i t}-\left.0.5 \rho w_{\infty}^{2}\left(r_{c r i t}\right) \cdot F_{1}\left(r_{c r i t}\right) C_{p, \min }\right|_{B E_{c r i t}} .
$$

Inserting this and $w_{\infty}\left(r_{c r i t}\right)$ from eq. (2.14) into eq. (2.42) eventually yields

$$
\sigma_{0.7, \text { crit }}=\frac{2 g r_{\text {crit }}}{c_{0}^{2}\left(1+(0.7 \lambda)^{2}\right)}-\left.\frac{\left((1-a)^{2}+\left(\frac{r_{c r i t}}{r_{\text {tip }}} \lambda\right)^{2}\left(1+a^{\prime}\right)^{2}\right)}{1+(0.7 \lambda)^{2}} F_{1}\left(r_{c r i t}\right) C_{p, \min }\right|_{B E_{\text {crit }}} .
$$

Evaluating $\sigma_{0.7, \text { crit }}$ for all values of $\lambda$ eventually provides the desired semi-empirical estimate of the turbine cavitation characteristics as in Fig. 1.

It is worth noting that, according to eq. $(2.47), \sigma_{0.7, c r i t}$ is not completely independent of the inflow velocity $c_{0}$. But this is a flaw of the fundamental definition of $\sigma_{0.7}$ - with the immersion depth of the turbine hub as a parameter.

\subsubsection{Hydrofoil Data}

Since lift and drag coefficients of the hydrofoil sections are functions of the Reynolds number

$$
R e=w_{\infty} l / v
$$

and the angle of attack, they are calculated in advance and stored in a database. These polar data is obtained utilizing the public domain code XFoil by DRELA [20]. In order to mimic a incompressible flow, the Mach number is set to zero. The transition from a laminar to turbulent boundary is calculated using the $e^{n}$-method with a threshold amplification ratio of $N_{\text {crit }}=1$, which corresponds to a free stream turbulence level of 2\% [21]. Post stall data are obtained from VITERNA et al. [22] - since empirical, not very accurate, but ensuring numerical stability. A visualized example of typical data is depicted in Fig. 7.

The chordwise pressure distribution required in the cavitation sub-model is also taken from XFoil. Since in the post stall region XFoil fails, we refrain from predicting post stall cavitation inception. 


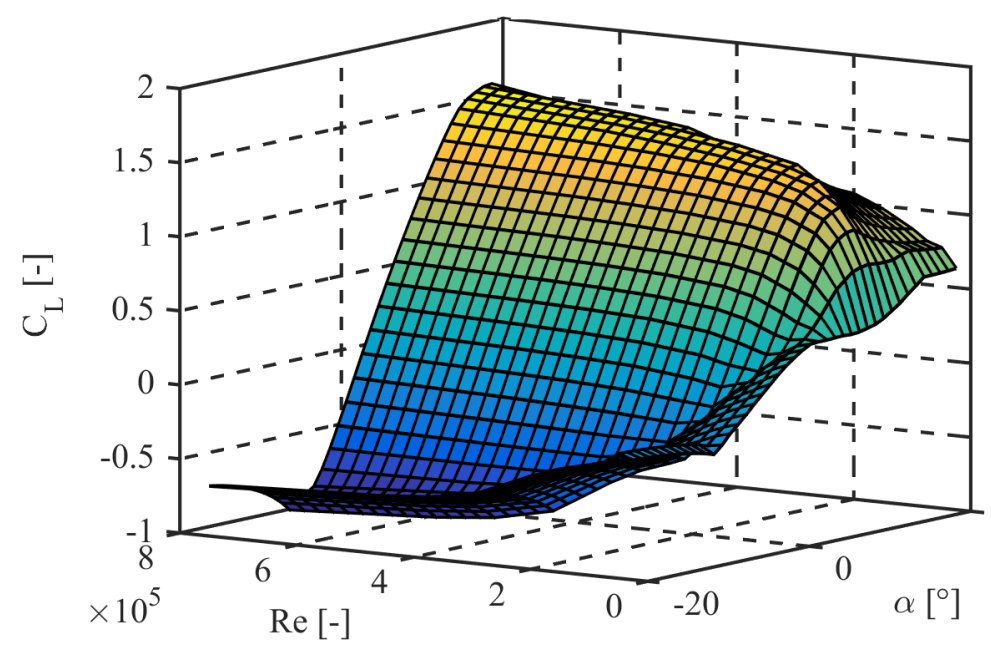

Fig. 7: Example: Lift coefficient $C_{L}$ of an own hydrofoil as a function of $R e$ and a and $\alpha$

\subsection{Solver}

While some literature suggests to solve the non-linear eqs. (2.23) and (2.24) with all their hidden supplementary model equations for $a$ and $a^{\prime}$ explicitly (e.g. [3]), we use an implicit solver, similar to the one reported by MASTERS et al. [12]. It was found that an implicit formulation avoids the numerical instabilities described in MANIACI [26]. A target function is set up as

$$
f\left(a, a^{\prime}\right) \equiv\left|C_{P, D F}-C_{P, M}\right|+\left|C_{T, D F}-C_{T, M}\right|
$$

and minimized via the NELDER-MEAD algorithm [24]. For an example set of parameters $\left(c_{0}=3 \mathrm{~m} / \mathrm{s}, \lambda=5, r / R\right.$ $=0.8)$ Fig. 8 shows $f\left(a, a^{\prime}\right)$. Evidently there is global minimum which ensures a unique solution. (There was no evidence of local minima as found by MASTERS et al. [12] for a similar error function -> löschen). Henceforth the criterion for convergence is set to $f=10^{-7}$.

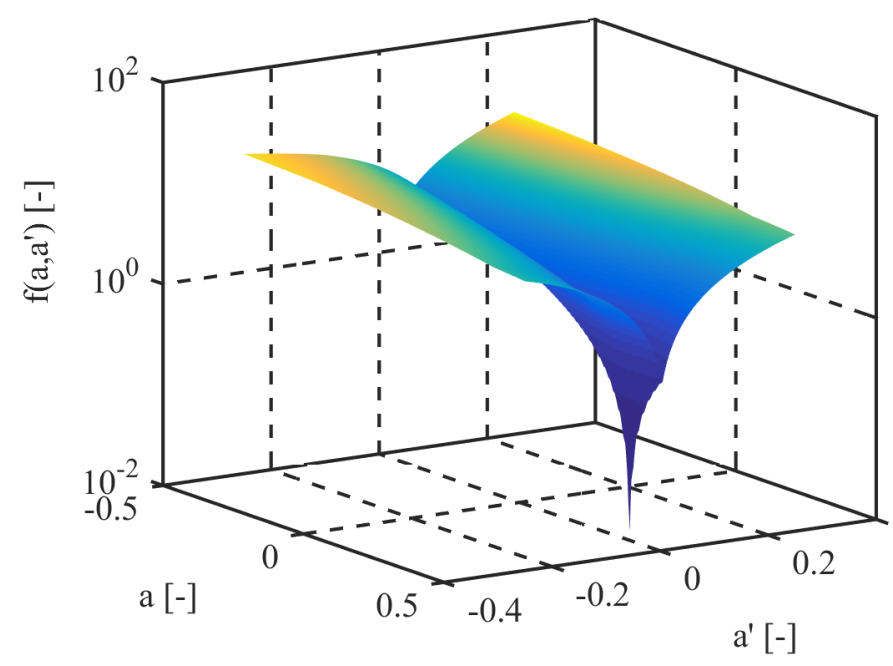

Fig. 8: Target function $f\left(a, a^{\prime}\right) \equiv\left|C_{P, B E}-C_{P, M}\right|+\left|C_{T, B E}-C_{T, M}\right|$ for an example set of parameters

\section{Validation}

An essential objective in this paper is the experimental validation of the performance prediction model presented above. Full scale experimental tests which yield power, thrust and cavitation characteristics with the usual accuracy of laboratory tests are unrealistic, even for comparably small turbines of a few meters of rotor size. Therefore we validate with two scaled models, one of a research turbine and one of an existing commercial. Results from the enhanced BEM are compared with published experimental data from a $1.5 \mathrm{~m}$ diameter turbine investigated at Queens University in Belfast [25-27] and - more comprehensively including cavitation inception - 
with new tests of an 1:8 scaled model of the $4 \mathrm{~m}$ diameter SCHOTTEL HYDRO Instream Turbine 250 (SIT250) turbine.

It is worth mentioning, that the usual flaw of model-scale tests, namely the lower Reynolds number as compared to full scale machines, is not of relevance here, since we predict the performance of the very same turbines as tested in the experiments.

\subsection{Validation Case 1: Queens University Turbine}

The Queens University Belfast tidal turbine, shown in Fig. 9, is a horizontal axis tidal turbine of $1.5 \mathrm{~m}$ diameter rotor size. It is well suited as a first validation case since a detailed description of the turbine geometry (foil shapes and blade characteristics) and the power take-off system has been published by JEFFCOATE et al. in [25] and [26] as well as $C_{P}(\lambda)$ characteristics ([25], [27]).

The turbine consists of a four bladed rotor and a nacelle, housing the drive train and generator. The nacelle is welded to a support strut. The true shaft torque and the angular speed were captured by an integrated torque sensor. The diameter of the nacelle and the fairing of the hub is $0.36 \mathrm{~m}$. The blades are built up from EPPLER E387 hydrofoil sections, the exact twist and chord distribution are given in [26]. The turbine performance was investigated experimentally through steady-state pushing tests in a lake; details of the testing procedure and results are published by JEFFCOATE et al. in [25]. The turbine was mounted on a catamaran type structure with a hub immersion depth of $1.5 \mathrm{~m}$, i.e. one rotor diameter. The test track in a narrow lake was approximately $400 \mathrm{~m}$ long, $30 \mathrm{~m}$ wide and $6 \mathrm{~m}$ deep with an effective blockage ratio less than $1 \%$. The inflow velocity profile was measured with an acoustic Doppler current profiler device and varied between 0.8 and 1.4 $\mathrm{m} / \mathrm{s}$. In a subsequent study, the turbine was tested in a large calm water towing tank at the Marine Technology Research Institute in Rome, Italy (CNR-INSEAN). Test procedure and results are presented by JEFFCOATE et al. in [26] and [27]. This towing tank is $460 \mathrm{~m}$ long, $13.5 \mathrm{~m}$ wide and $6 \mathrm{~m}$ deep which results in a blockage ratio (cross-sectional) of $2 \%$. Initially the turbine was installed with a hub depth of $1.5 \mathrm{~m}$. Subsequent studies with a varying immersion depth are not of interest for this work. The inflow velocity (carriage speed) had been varied from $0.8 \mathrm{~m} / \mathrm{s}$ to $1.4 \mathrm{~m} / \mathrm{s}$ corresponding to the initial pushing tests in the lake.

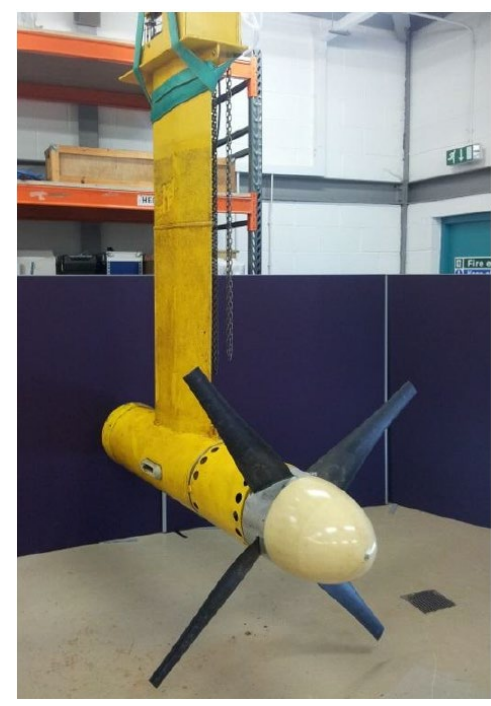

Fig. 9: Tidal turbine of the Queens University Belfast (QUB) [27]

Using the BEM model described above, the $C_{P}(\lambda)$ characteristics of this turbine for inflow velocities 0.8 $\mathrm{m} / \mathrm{s}$ and $1.4 \mathrm{~m} / \mathrm{s}$ are predicted, Fig. 10. The effect of inflow velocity and hence Reynolds number is barely visible, the collapse of the curves is nearly perfect. For comparison the left diagram in Fig. 10 shows measurements from [25] (taken from turbine 2 in the original paper) and on the right side from [27]. Given the scatter of the experimental data the overall agreement is very good. It should be mentioned that at low tip speed ratios $(0<\lambda<1.5)$ the blades are stalled. Naturally, lift and drag data for the hydrofoil sections are somewhat uncertain (see 2.2.4). At higher tip speed ratios $(\lambda>4)$ the BEM overpredicts the power coefficients. An explanation is that, as mentioned in [25], a bending of the blades occurs at higher tip speed ratios, leading to a decrease of $C_{P}$. By contrast, in the BEM prediction we assume absolutely rigid blades.

Based on the pushing tests also he thrust characteristic is reported in [25]. Unfortunately the measured values were normalized by the maximum of a 4 th order polynomial curve fit. Hence, for a comparison, our BEM predictions are normalized using the maximum thrust coefficient $C_{T, \max }=0.42$ at $\lambda=2.9$, which, of course, 
allows a comparison of the shape but not of the absolute values of the thrust characteristic, Fig. 11. At low tip speed ratios $(\lambda<2.6)$, the BEM tool underpredicts the measured thrust coefficients significantly. The deviations again are attributed the inaccurate polar data for the stalled airfoil sections and the inherently transient flow in this operation regime, which is not covered by the BEM theory. At higher tip speed ratios, however, the agreement between the experimental data and the BEM predictions are considered very satisfying.
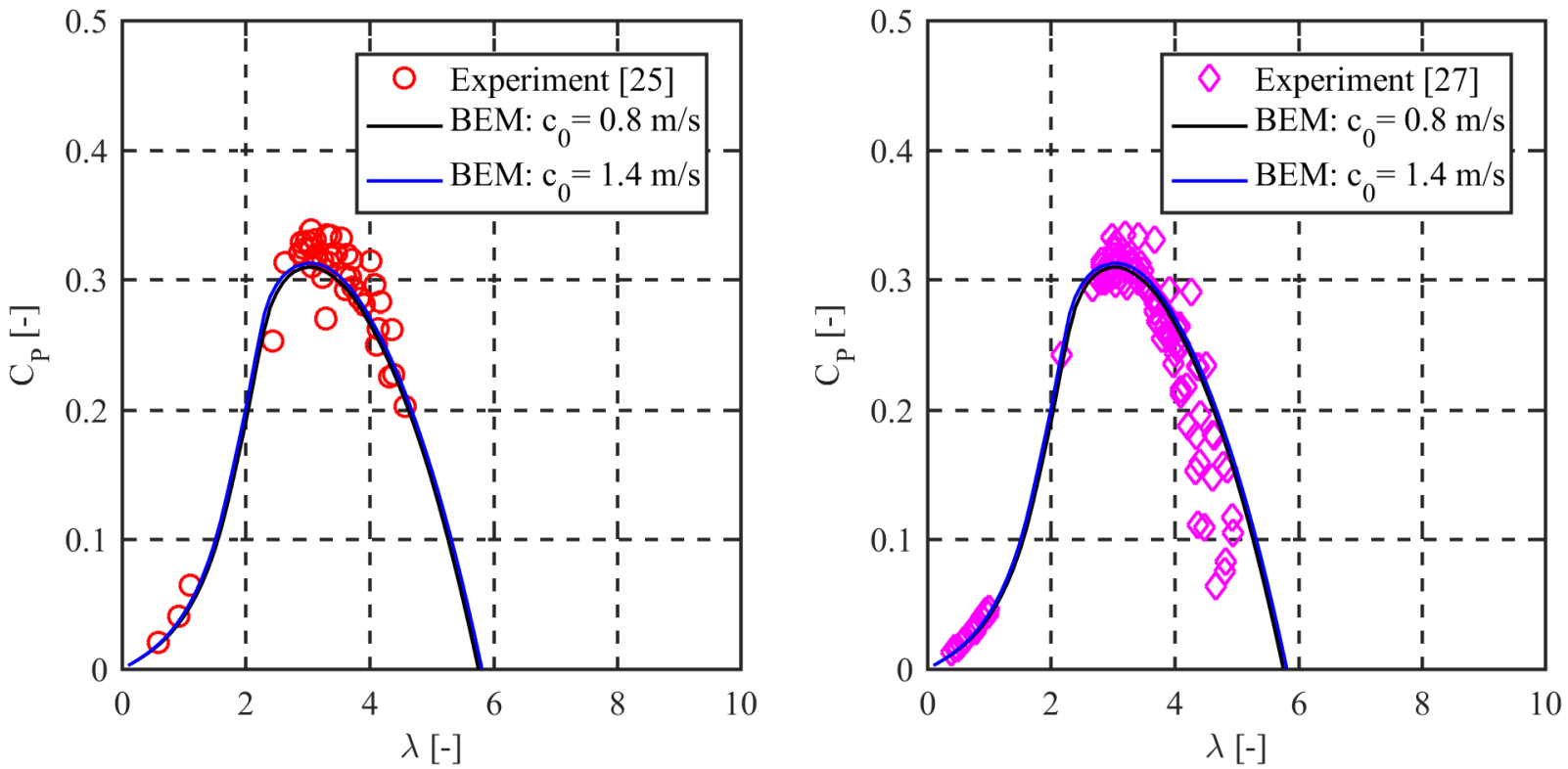

Fig. 10: Queens University Belfast tidal turbine: BEM-predicted power coefficient $C_{P}$ vs. tip speed ratio $\lambda$ and comparison with experimental data from [25] (left) and [27] (right)

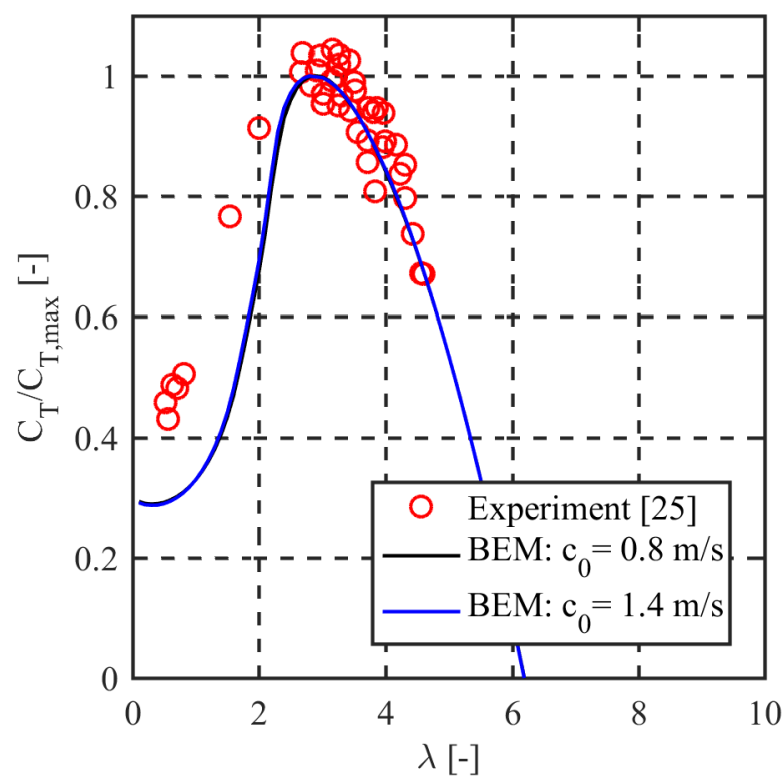

Fig. 11: Queens University Belfast tidal turbine: BEM-predicted normalized thrust coefficient $C_{T} / C_{T, \max }$ vs. the tip speed ratio $\lambda$ and comparison with experimental data from [25]

\subsection{Validation Case 2: University of Siegen/Schottel Turbine}

The University of Siegen/Schottel turbine is commercially available as the SCHOTTEL HYDRO Instream Turbine 250 (SIT250), Fig. 12. It is a three-bladed fixed-pitch turbine with a design tip-speed ratio $\lambda=5$. The rotor diameter of the 1:8 scale model, which was tested here exclusively, is $0.5 \mathrm{~m}$. It is CNC-milled from brass. Since this turbine was originally designed by the first author of this paper, the full geometry of all blade elements forming the blade is known in detail. All model scale experiments were performed at the Schiffbau-Versuchsanstalt Potsdam GmbH (SVA Potsdam). The most relevant data of the towing tank are compiled in Table 4.1. The shaft of the turbine is immersed one rotor diameter below the waterline. The cross-sectional blockage of the 
open water tests was $0.5 \%$. Fig. 13 depicts the towing tank setup. For cavitation studies the turbine is placed in a cavitation tunnel with a specification as in Table 4.2.
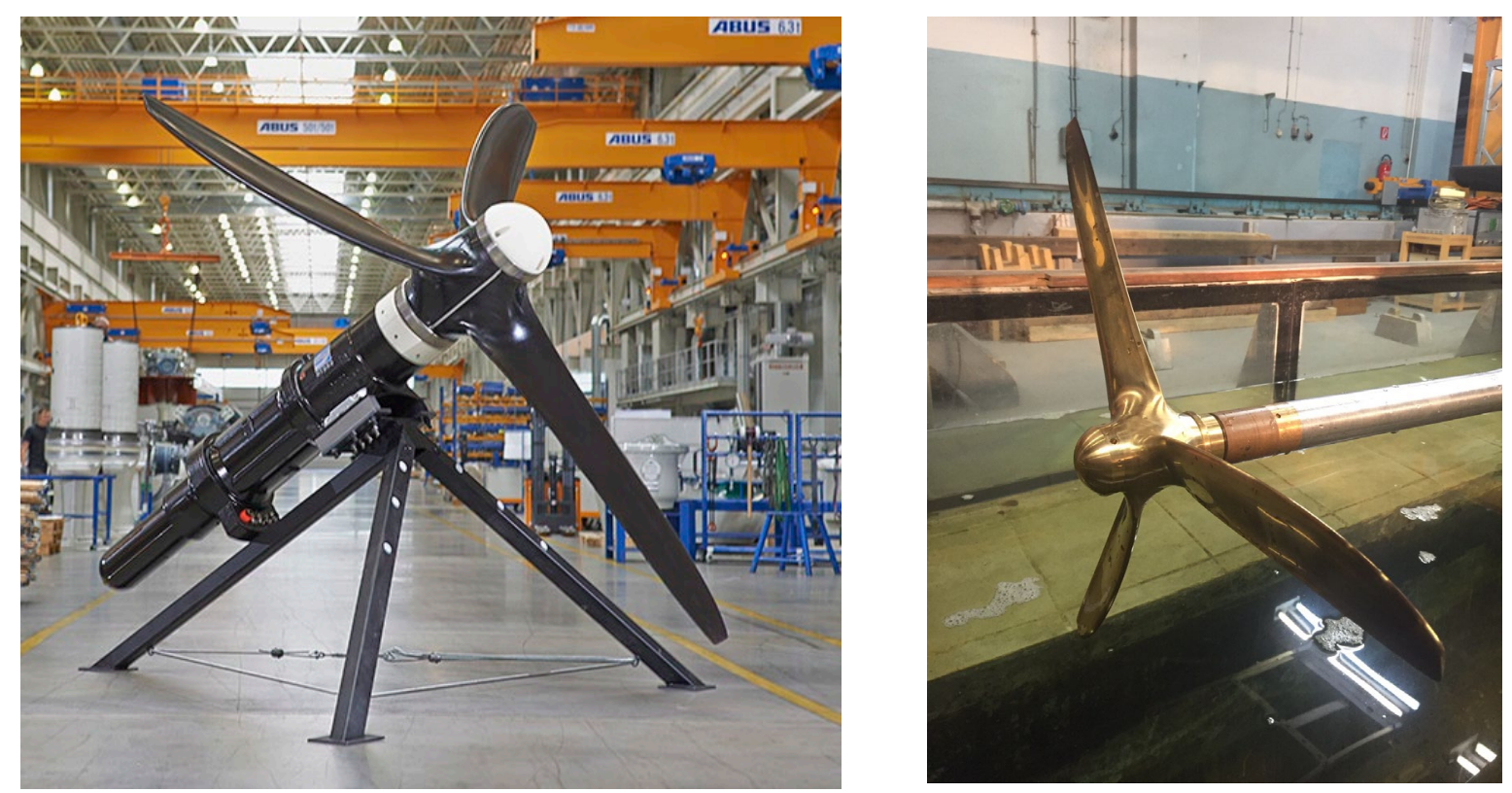

Fig. 12: Full-scale SCHOTTEL SIT250 (left) and the 1:8 scaled brass model for the validation experiments (right)

Table 4.1: SVA towing tank test facility

\begin{tabular}{|l|l|}
\hline Towing tank dimensions & $280.0 \mathrm{~m}$ length \\
& $9.0 \mathrm{~m}$ width \\
& $4.5 \mathrm{~m}$ depth \\
\hline Maximum carriage velocity (corresponding to $\left.c_{0}\right)$ & $7.0 \mathrm{~m} / \mathrm{s}$ \\
\hline Dynamometer type H39, Kempf and Remmers & $n_{\max }=60 \mathrm{rmp}$ \\
& $T_{\max }=1000 \mathrm{~N}$ \\
& $Q_{\max }=50 \mathrm{Nm}$ \\
\hline Shaft immersion depth & $0.5 \mathrm{~m}$ \\
\hline
\end{tabular}

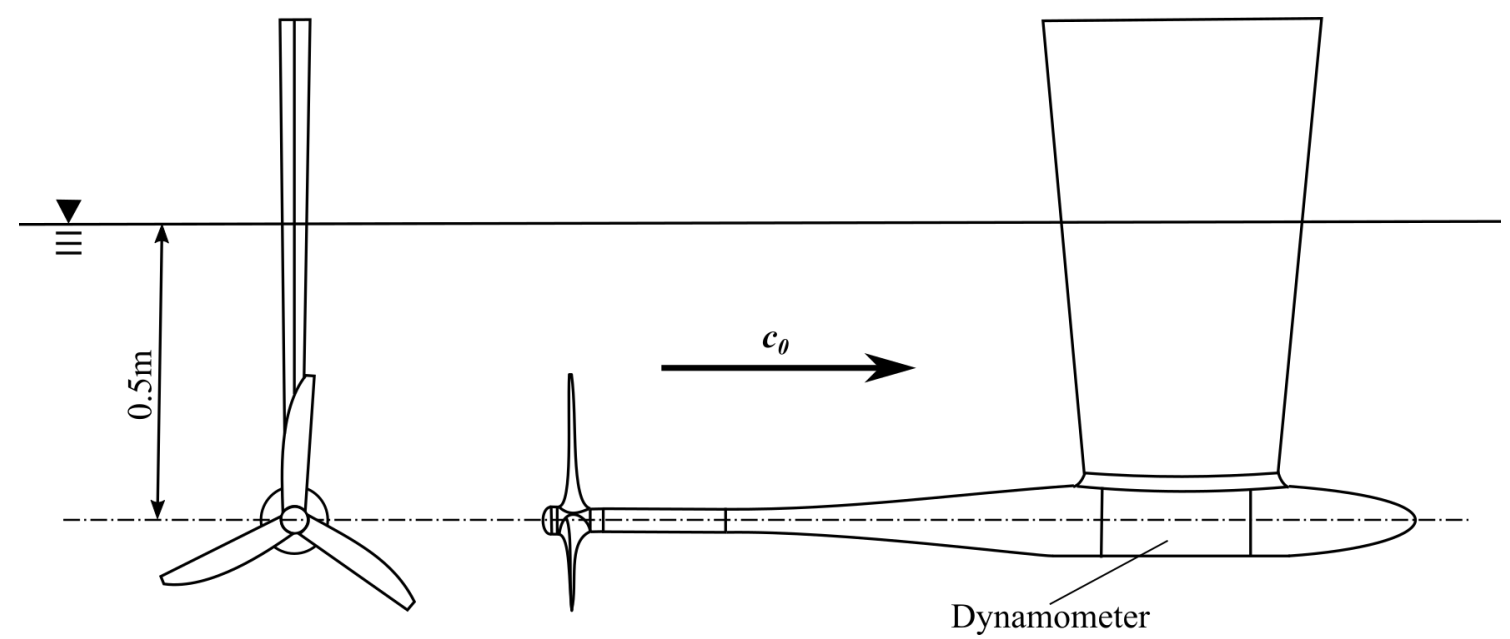

Fig. 13: SVA towing tank setup

Table 4.2. SVA Cavitation test facility

\begin{tabular}{|l|l|}
\hline Cavitation tunnel test section (length) & $2600 \mathrm{~mm}$ \\
\hline Cavitation tunnel test section (cross section) & $850 \mathrm{~mm} \times 850 \mathrm{~mm}$ \\
\hline
\end{tabular}




\begin{tabular}{|l|l|}
\hline Pressure variation & $950 \mathrm{mbar}-1200 \mathrm{mbar}$ \\
\hline Maximum flow velocity (corresponding to $c_{0}$ ) & $7.5 \mathrm{~m} / \mathrm{s}$ \\
\hline Dynamometer H36, Kempf and Remmers & $n_{\max }=60 \mathrm{~s}-1$ \\
& $T_{\max }=2000 \mathrm{~N}$ \\
& $Q_{\max }=100 \mathrm{Nm}$ \\
\hline
\end{tabular}

The basic testing procedure in the towing tank and the cavitation tunnel is the same. Tests were carried out at three different velocities $\left(c_{0}=2.0,2.5,3.0 \mathrm{~m} / \mathrm{s}\right)$ and over a range of tip speed ratios, which had been achieved by varying the rotational speed of the turbine for a given inflow velocity. $10 \mathrm{~s}$-time records of thrust, torque, rotational speed and inflow speed were recorded synchronously, allowing an assessment of accuracy. According to the recommendations of the International Towing Tank Conference (ITTC) [28], the measured values for thrust and torque were corrected using a dummy hub having the same mass as the model turbine.

The cavitation inception tests were carried out from $\lambda=4.4$ to $\lambda=8.9$, following the ITTC standards for cavitation tests [29]. Due to the high cross-sectional blockage ratio of $27 \%$, a blockage correction had to be applied in the cavitation tunnel to match the operation points at free flow conditions. Two different inflow velocities $\left(c_{0}=2 \mathrm{~m} / \mathrm{s}\right.$ and $\left.c_{0}=3 \mathrm{~m} / \mathrm{s}\right)$ were necessary to cover the desired wide range of operating conditions. Hence, the cavitation number characteristic was obtained by adjusting the inflow velocity, the rotational speed of the turbine and the static pressure inside the test section. Cavitation inception was triggered by decreasing the static pressure, coming from a non-cavitating condition. Cavitation inception was identified visually.

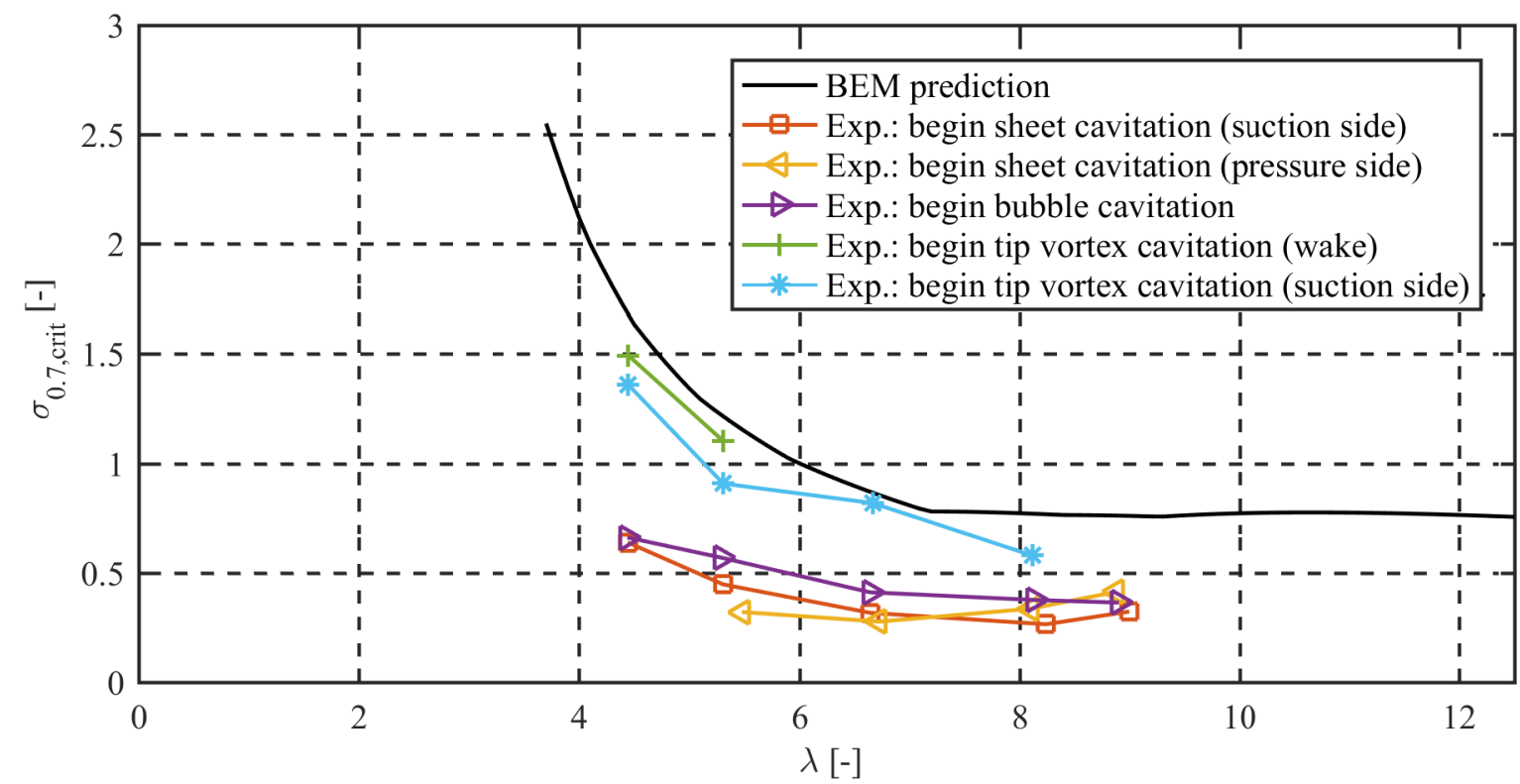

Fig. 14: 1:8 scaled model of the University of Siegen/Schottel Turbine: Predicted critical cavitation number vs. tip speed ratio $\lambda$ and comparison with the experimental data.

The experimentally obtained cavitation inception numbers of various cavitation types are plotted versus the tip speed ratio in Fig. 14 and compared to the BEM predicted curve of the critical cavitation number $\sigma_{0.7, \text { crit }}$. The dependency of $\sigma_{0.7, \text { crit }}$ on $c_{0}$ is negligible for the presented cases, hence, the $\sigma_{0.7, \text { crit }}$ curve presented is valid for all inflow velocities considered. The order of occurrence of the different cavitation types is obvious: Tip vortex cavitation - vortex cavitation on the suction side - bubble cavitation and sheet cavitation on suction and pressure side. As discussed in section 2.2.3, the turbine cavitation inception number $\sigma_{0.7, c r i t}$ marks the maximum cavitation number for which the local static pressure on a BE equals the vapor pressure, not taking into account tip vortex phenomena. The BEM model overpredicts somewhat the experimentally identified cavitation inceptions. However, one has to keep in mind, that in the experiments the onset of cavitation is based purely on visual observation. Vaporization may occur earlier not visible to the naked eye. This can explain the differences. In any case the predicted $\sigma_{0.7, \text { crit }}$ is a conservative estimate of cavitation inception, irrespective of the cavitation type.

In the upper part of Fig. 15, the characteristic power curves, obtained from the towing tank tests, are compared for different inflow velocities $c_{0}$. Chord Reynolds numbers for the $2 \mathrm{~m} / \mathrm{s}$ inflow velocity along the span range from about $3 \cdot 10^{4}$ to $5 \cdot 10^{5}$, and for $3 \mathrm{~m} / \mathrm{s}$ from $4.5 \cdot 10^{4}$ to $9.5 \cdot 10^{5}$. As expected the $C_{P}$ values are increasing with the inflow velocity due to the higher Reynolds numbers - with one exception: For $\lambda>8.1$ the $c_{0}=$ $3 \mathrm{~m} / \mathrm{s}$ curve undercuts the $c_{0}=2 \mathrm{~m} / \mathrm{s}$ curve. This can only be explained by the onset of cavitation. 

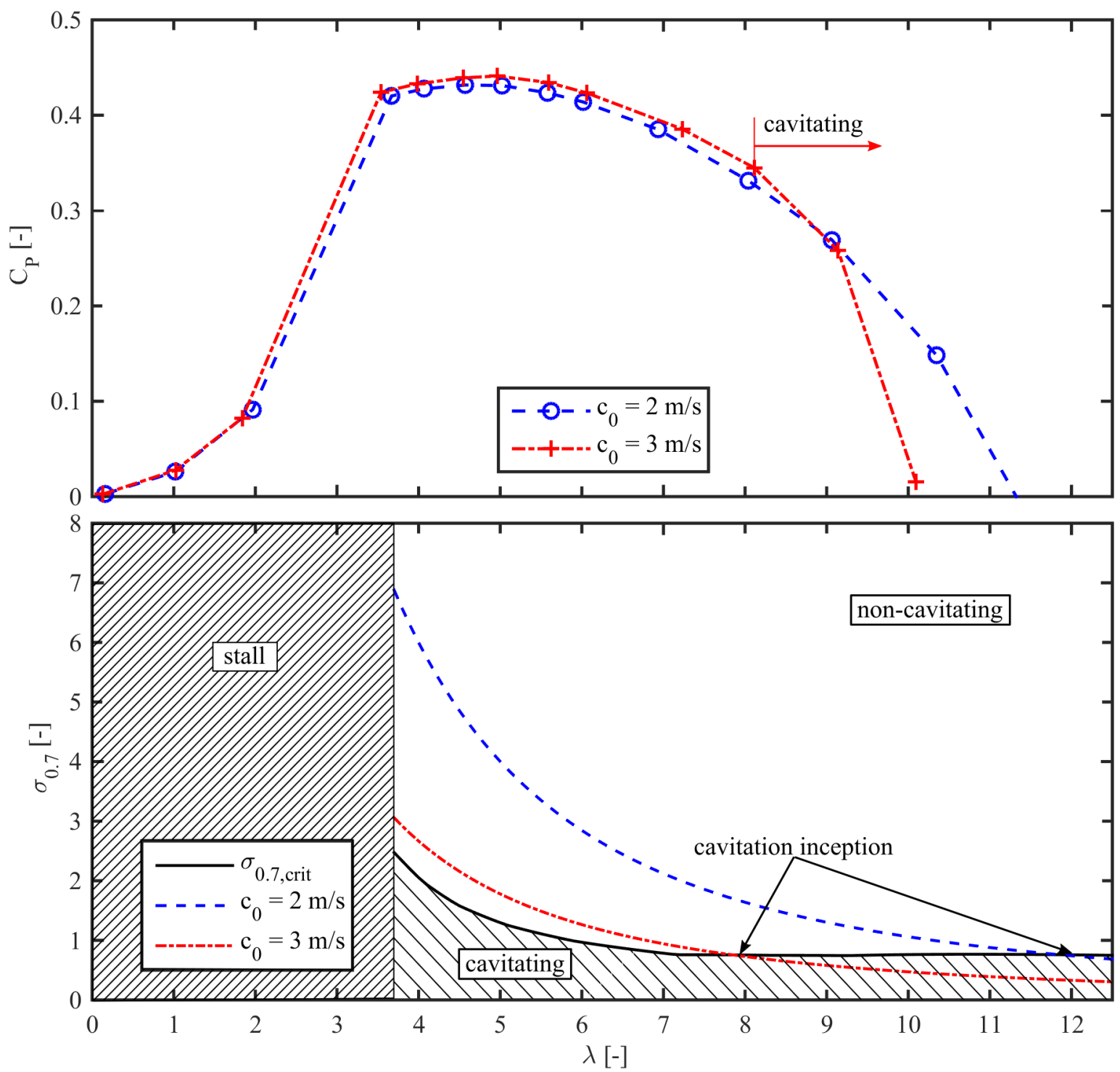

Fig. 15: 1:8 scaled model of the University of Siegen/Schottel turbine; upper: Measured power coefficient $C_{P}$ vs. tip speed ratio $\lambda$ for different inflow velocities $c_{0}$; lower: BEM-predicted critical cavitation number $\sigma_{0.7, c r i t}$ Vs. tip speed ratio $\lambda$ and comparison with operational cavitation numbers $\sigma_{0.7}$ of the towing tests at different inflow velocities $c_{0}$.

The lower diagram in Fig. 15 shows the operational cavitation number $\sigma_{0.7}$ plotted versus tip speed ratio for the inflow velocities of the towing tank tests. Additionally, the predicted critical cavitation number $\sigma_{0.7, \text { crit }}$ is shown. Stalling operating points are excluded, since the cavitation prediction model is not valid for these conditions (see section 2.2.3). Cavitation at the turbine blades is identified for operating points where $\sigma_{0.7} \leq$ $\sigma_{0.7, \text { crit. }}$. Hence, a load reduction due to cavitation is predicted for $\lambda \leq 7.9\left(c_{0}=3 \mathrm{~m} / \mathrm{s}\right)$ and $\lambda \leq 12\left(c_{0}=2 \mathrm{~m} / \mathrm{s}\right)$, respectively. This is in a good agreement with the observation shown in the upper diagramm of Fig. 15, i.e. the model predicts the inception of performance reducing cavitation with satisfactory accuracy.

In Fig. 16 the characteristic power and thrust curves, obtained from the towing tank tests, are compared with the BEM predictions for two different inflow velocities $c_{0}$. Since a load correction for cavitating conditions is not included in the BEM model, the BEM predictions are solely presented for non-cavitating operating points. For these operating points the predicted and experimentally obtained power coefficients show a good agreement. The predicted and measured thrust coefficients differ significantly for tip speed ratios below 3.7. This is attributed to the inherent weakness of the VITERNA approximation of lift and drag of a stalled airfoil section. At $3.7 \leq \lambda$ the $C_{T}$ values from BEM are in good agreement with the experimental data for both inflow velocities. 

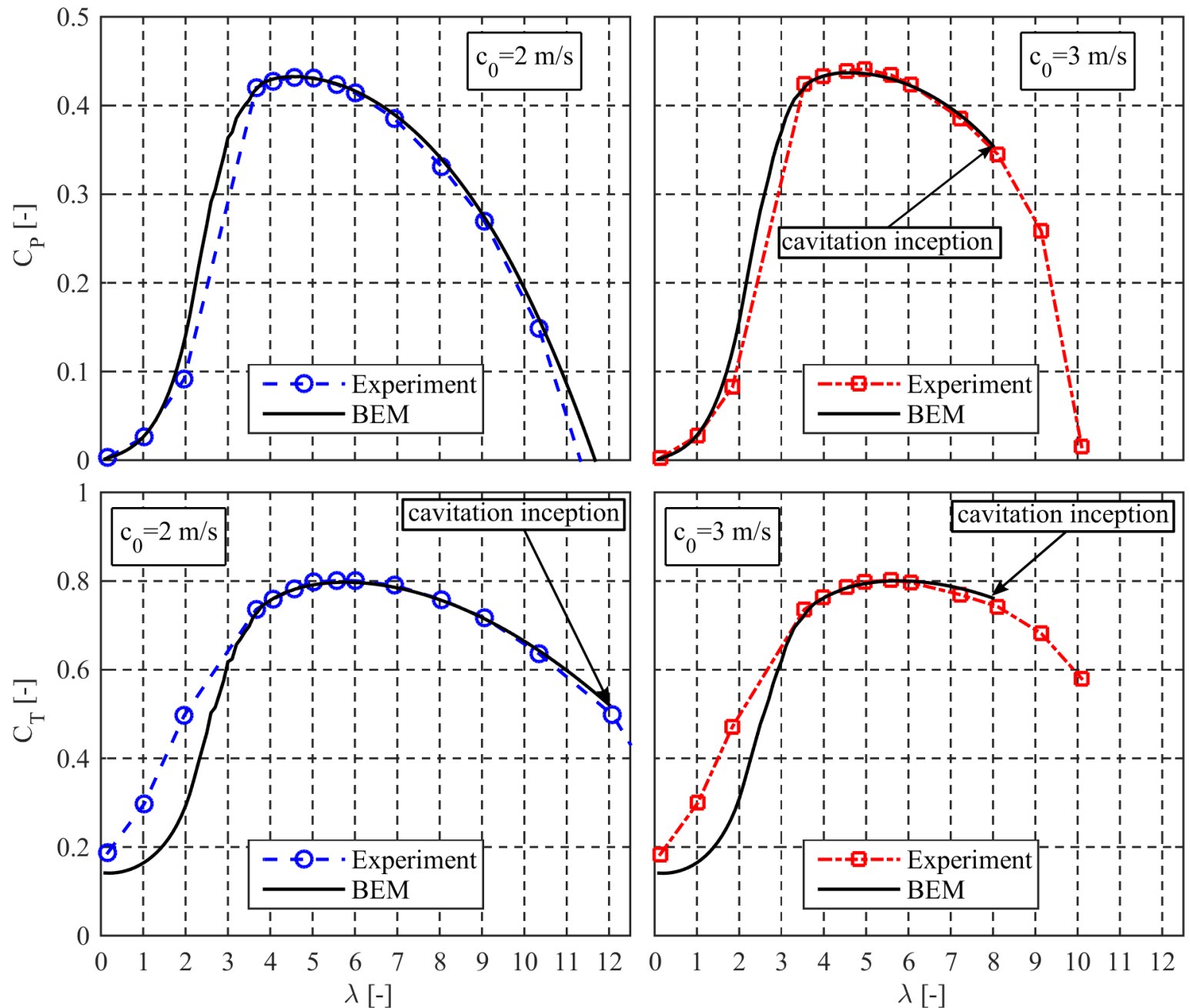

Fig. 16: 1:8 scaled model of the University of Siegen/Schottel turbine; upper: BEM-predicted power coefficient $C_{P}$ vs. tip speed ratio $\lambda$ and comparison with experimental data; lower: BEM-predicted thrust coefficient $C_{T}$ vs. tip speed ratio $\lambda$ and comparison with experimental data.

The left graph of Fig. 17 once more illustrates the effect SHEN's tip loss factor $F_{1}$. While the uncorrected $\left.C_{p, \min }\right|_{\mathrm{BE}}$ values increase within monotonically towards the tip, the correction forces $\left.C_{p, \min }\right|_{\mathrm{BE}}$ to zero at the blade tip. On the right side of Fig. 17, the predicted critical cavitation number $\sigma_{0.7, \text { crit, }}$ with and without correction are plotted versus the tip speed ratio and compared to the results from the cavitation tunnel (excluding tip vortex cavitation). As a conclusion the correction of the near tip suction peaks leads to a significant improvement of the $\sigma_{0.7, \text { crit }}$ prediction.

As mentioned above, the predicted critical cavitation number shown in Fig. 14 contains no information of the cavitation type and its location on the blade. Therefore calculated flow kinematics from the BEM and the pressure coefficient data of the blade elements where used to calculate the pressure distribution on the blade. By calculating the pressure difference $\Delta p=p_{\text {stat }}-p_{v}$ for the complete blade, the locations where cavitation is assumed, can be identified. A photograph of the suction side at $\lambda=4.4$ and $\sigma_{0.7}=0.639$ is shown on the left side of Fig. 18. The radial distances of $r / r_{t i p}=0.5,0.7,0.8,0.9$ and 0.95 are tagged by black stripes. On the right side of Fig. 18, the predicted $\Delta p$ - distribution is shown. While $\Delta p<0$ is predicted close to the leading edge from ca. $r / r_{t i p}=0.35$ to the tip, cavitation can only be detected visually between approximately $60 \%$ and ca. $77 \%$ of the blades span, with a larger distance to the leading edge. As already discussed this suggests, that cavitation is not necessarily visible, when the static pressure drops below the vapor pressure. 

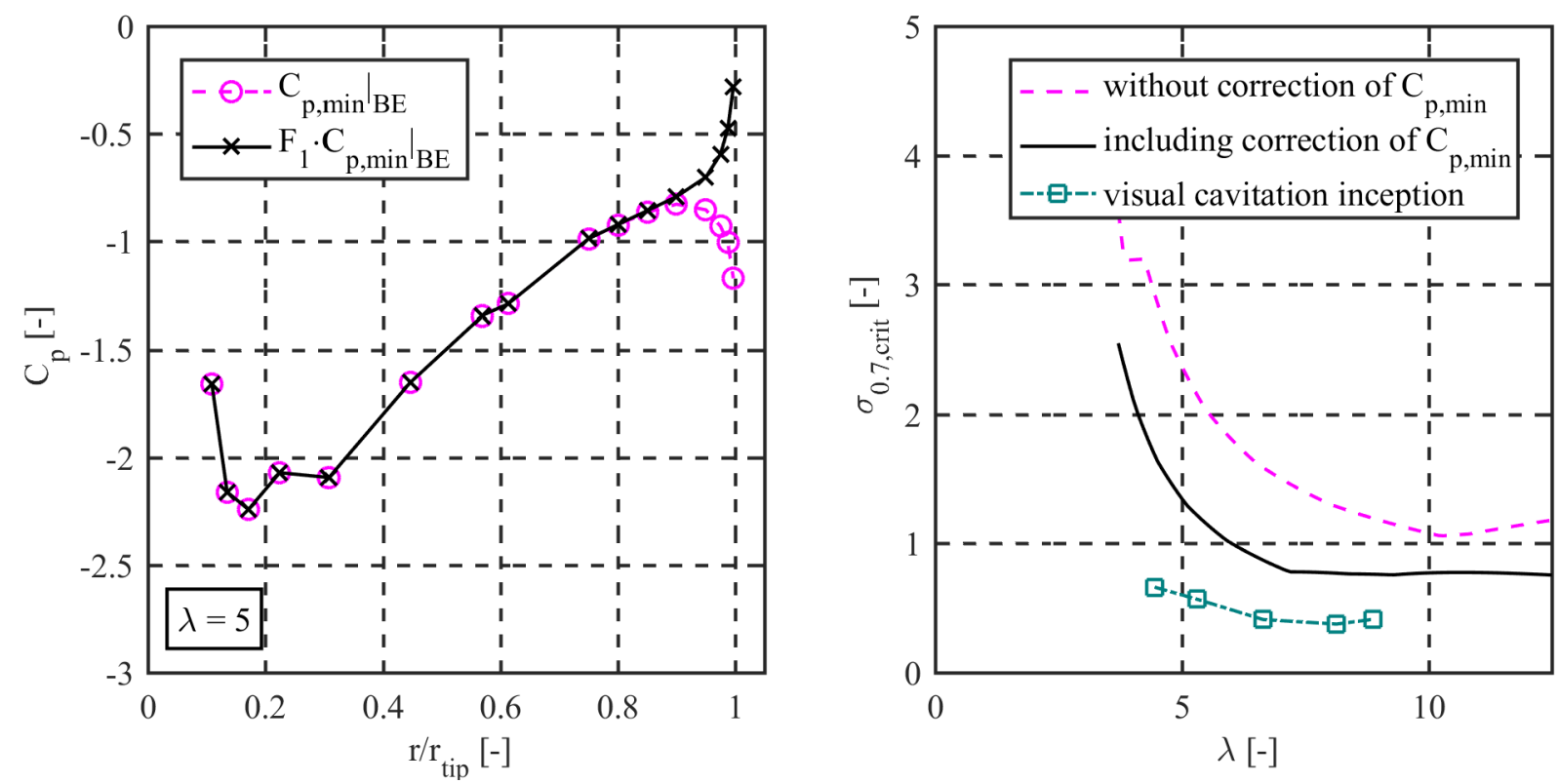

Fig. 17: 1:8 scaled model of the University of Siegen/Schottel turbine; Left: Uncorrected and corrected minimum pressure coefficient of BEs along the span at $\lambda=5$ and $c_{0}=2 \mathrm{~m} / \mathrm{s}$. Right: BEM predicted critical cavitation number $\sigma_{0.7, c r i t}$ Vs. tip speed ratio $\lambda$ with and without correction of the suction peaks $C_{p \text {, min }}$ compared to the experimentally obtained visual cavitation inception.
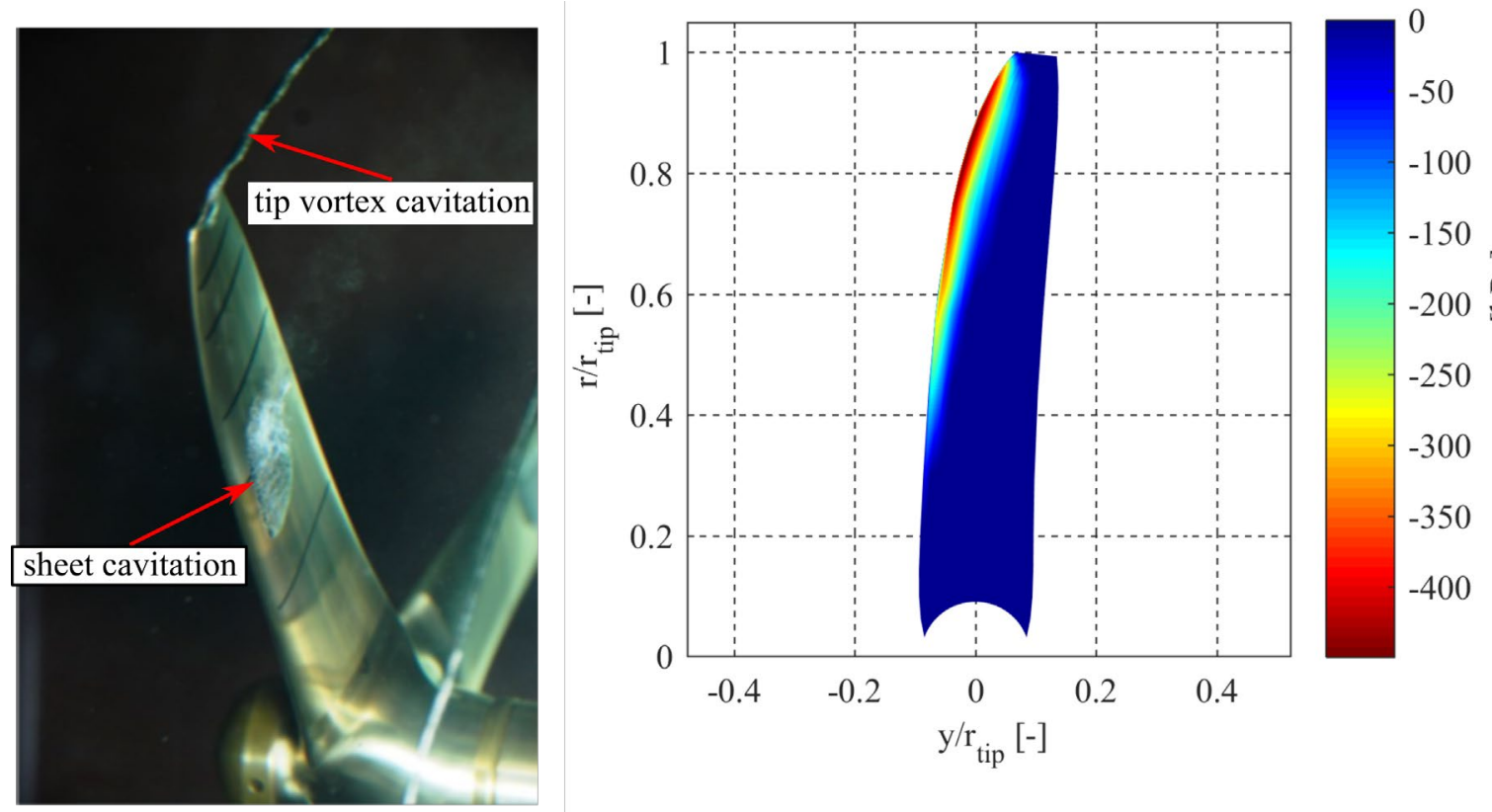

Fig 18: 1:8 scaled model of the University of Siegen/Schottel turbine; Left: Photograph of cavitating blade (tip cavitation and suction side sheet cavitation) at $\lambda=4.4$ and $\sigma_{0.7}=0.639$; right: BEM prediction of $p_{\text {stat }}-p_{v}<0$ on the suction side at the same operating point.

\section{Summary and Conclusions}

An enhanced semi-analytical model has been developed that allows the prediction of the performance characteristics including cavitation inception of horizontal axis tidal turbines. A central component is the wellknown blade element momentum theory which is refined by various submodels. For instance, airfoil section lift and drag are determined for the local Reynolds number and angle of attack; BUHL's thrust coefficient correction is used for large axial induction factors, and models by PRANDTL/GLAUERT and SHEN account for blade hub and tip losses. Flow kinematics and the static pressure distribution on the blade surface are the inputs in a criterion for cavitation inception, the cavitation number. In order to avoid numerical instabilities, an implicit solver utilizing the NELDER-MEAD algorithm is suggested to solve the complete set of non-linear equations. 
A key objective of this paper was the comprehensive experimental validation of the model. For that experimental data from a tidal turbine, developed by the Queens University Belfast, and from a 1:8 model of the SCHOTTEL HYDRO turbine SIT250 were compared with performance predictions. Predicted power and thrust coefficient characteristics were found to agree well with the experimental results for a wide operational range and different inflow velocities. Discrepancies were observed only at low tip speed ratios where major parts of the blades operate under stall conditions. The predicted critical cavitation number is somewhat larger than the measured, i.e. the prediction is conservative. One possible reason for this discrepancy is the fact that in the experiments cavitation inception was identified by visual observation only. Therefore, e.g. acoustic signatures will be checked instead in future studies. It turned out that taking into account hydraulic blade tip effects in the model enhances the precision of the predicted critical cavitation number substantially.

As an overall conclusion the semi-analytical model developed seems to be so fast, accurate and robust that it can be integrated in a future workflow for optimizing tidal turbines. Although we targeted at small to medium size fixed pitch turbines, it even may be applied to larger turbines.

\section{Acknowledgements}

This work has been funded by the Federal Ministry for Economic Affairs and Energy of Germany (BMWi) in the project TIDAL POWER (FKZ 0325817B). The results of the model scale test were provided by SCHOTTEL HYDRO GmbH.

\section{$5 \quad$ References}

[1] Kumar, P., Saini, R. P.: Study of cavitation in hydro turbines - A review. Renewable and Sustainable Energy Reviews 14 (2010) 1, 374-383

[2] Gasch, R.: Windkraftanlagen. Grundlagen, Entwurf, Planung und Betrieb. Stuttgart: Springer Vieweg 2013

[3] Hansen, M. O. L.: Aerodynamics of Wind Turbines. London: Earthscan 2007

[4] Burton, T.: Wind energy. Handbook. Chichester, New York: J. Wiley 2001

[5] Gerhard, T., Carolus, T.: Small horizontal axis wind turbine: Analytical blade design and comparison with RANS-prediction and first experimental data. Proceedings of the ASME Turbo Expo: Turbine Technical Conference and Exposition - 2013. Presented at the 2013 ASME Turbo Expo: Turbine Technical Conference and Exposition; June 3 - 7, 2013, San Antonio, Texas, USA. New York, N.Y: ASME 2013

[6] NWTC Information Portal (AeroDyn). https://nwtc.nrel.gov/AeroDyn. Last modification July 27, 2016; Accessed September 8, 2016

[7] Glauert, H.: Airplane propellers. In:Durand, W. F. (ed.): Aerodynamic Theory, Springer, Berlin (1935), 169-360

[8] Spera, D. A.: Wind turbine technology. Fundamental concepts of wind turbine engineering. New York: ASME Press 1994

[9] Buhl, M. L.: New Empirical Relationship between Thrust Coefficient and Induction Factor for the Turbulent Windmill State. NREL/TP-500-36834. Golden, CO: National Renewable Energy Laboratory, 2005

[10] Shen, W. Z., Mikkelsen, R., Sørensen, J. N., Bak, C.: Tip loss corrections for wind turbine computations. Wind Energy 8 (2005) 4, 457-475

[11] Batten, W., Bahaj, A. S., Molland, A. F., Chaplin, J. R.: The prediction of the hydrodynamic performance of marine current turbines. Renewable Energy vol. 33 (2008) 5, S. 1085-1096

[12] Masters, I., Chapman, J. C., Wilis, M. R., Orme, J.: A Robust Blade Element Momentum Theory Model for Tidal Stream Turbines Including Tip and Hub Loss Corrections. Journal of Marine Engineering and Thechnology (2011) 10(1): 25-35

[13] Molland, A. F., Bahaj, A. S., Chaplin, J. R.,Batten, W. M. J.: Measurements and predictions of forces, pressures and cavitation on 2-D sections suitable for marine current turbines. Proceedings of the Institution of Mechanical Engineers, Part M: Journal of Engineering for the Maritime Environment 218 (2004) 2, $127-138$

[14] Batten, W., Bahaj, A. S., Molland, A. F. Chaplin, J. R.: Hydrodynamics of marine current turbines. Renewable Energy 31 (2006) 2, 249-256

[15] J. Baltazar, J. Machado, J. A. C. Falcao de Campos: Hydrodynamic design and analysis of horizontal axis marine current turbines with lifting line and panel methods. ASME $201130^{\text {th }}$ International Conference on Ocean, Offshore and Arctic Engineering. 2011, 453-465

[16] Bal, Ş., Altar, M., Uşar, D.: Performance prediction of horizontal axis marine current turbines. Ocean System Engineering, vol. 5, No. 2, 125-138, 2015

[17] Moriarty, P. J.; Hansen, A. C.: AeroDyn theory manual. Golden, CO: National Renewable Energy Laboratory, 2005 
[18] Shen, W Z, Zhu, W. J., Sørensen, J. N: Study of tip loss corrections using CFD rotor computations. Journal of Physics: Conference Series, vol. 555, Conference 1, 2014

[19] Schiffbau-Versuchsanstalt Potsdamm: Turbine tests with the model turbine TR0044 in the towing tank and the cavitation tunnel. Unpublished Report 4442. Potsdam, 2016

[20] Drela, M.: XFoil - Subsonic Airfoil Development System, 2013. Available: http://web.mit.edu/drela/Public/web/xfoil/. Accessed: August 28, 2016

[21] Drela, M.: XFOIL: An Analysis and Design System for Low Reynolds Number Airfoils. In: Low Reynolds Number Aerodynamics. Proceedings of the Conference Notre Dame, Indiana, USA, 5-7 June 1989. Lecture Notes in Engineering, Bd. 54. Berlin, Heidelberg: Springer 1989, 1-12

[22] Viterna, L. A., Corrigan, R. D.: Fixed pitch rotor performance of large horizontal axis wind turbines. DOE/NASA workshop on large HAWTs. Cleveland, Ohio, 1981

[23] Maniaci, D.: An Investigation of WT_Perf Convergence Issues. 49 ${ }^{\text {th }}$ AIAA Aerospace Sciences Meeting including the New Horizons Forum and Aerospace Exposition, 2011

[24] Nelder, J. A., Mead, R.: A Simplex Method for Function Minimization. The Computer Journal 7 (1965) 4 , 308-313

[25] Jeffcoate, P., Elsaesser, B., Whittaker, T and Boake, C.: Field tests of multiple 1/10 scale tidal turbines in steady flows. Renewable Energy, vol. 87, 240-252, 2016.

[26] Jeffcoate, P., Salvatore, F., Boake, C., Elsaesser, B.: Effect of submergence on tidal turbine performance. Paper Presented at EWTEC, Nantes France, September 6-11, 2015.

[27] Jeffcoate, P., Elsaesser, B.: Infrastructure Access Report - Invest Northern Ireland tandem tidal turbines project - towing tank test. 2014. Available: http://www.marinet.eu/public/docs/INITTT_CNRINSEAN Infrastructure Access_Report.pdf . Accessed: September 30, 2016.

[28] ITTC-Recommended Procedures - testing end extrapolating methods, propulsion, propulsor, open water tests. 2002. Available: http://ittc.info/media/2039/75-02-03-021.pdf . Accessed September 28, 2016

[29] ITTC-Recommended Procedures and Guidelines- testing end extrapolating methods, propulsion, cavitation, model-scale cavitation test. 2002. Available: http://ittc.info/media/1844/75-02-03-031.pdf . Accessed September 28, 2016 